\title{
Finite-time event-triggered control and fault detection for singular Markovian jump mixed delay systems under asynchronous switching
}

\author{
Mengzhuo Luo ${ }^{1 *}$, Shouming Zhong ${ }^{2}$ and Jun Cheng ${ }^{3}$
}

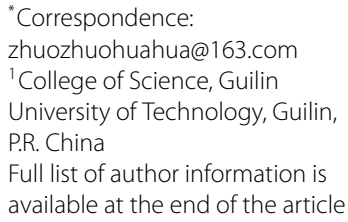

\section{空 Springer}

\begin{abstract}
This paper considers the problem of the simultaneous finite-time event-triggered control and fault detection for a class of continuous-time singular Markovian jump mixed delay systems (SMJDSs) under asynchronous switching. In order to develop the control and detection objectives, the mode-dependent fault detection filters and dynamic feedback event-triggered-based controllers are designed and the switching signal between the detector/controller unit and subsystems is assumed to be asynchronous. Based on average dwell time (ADT) techniques, some new sufficient conditions for the existence of fault detection/controller unit are presented in the framework of linear matrix inequalities (LMIs) to ensure the control system has singular stochastic finite-time stability (SSFTS). Finally, a numerical example is provided to illustrate the effectiveness of the proposed method.
\end{abstract}

Keywords: Detector/controller unit; Event-triggered control and fault detection; Singular stochastic finite-time stability; Asynchronous switching; Average dwell time

\section{Introduction}

In the past decade, the fault detection problem has been widely investigated due to the rising demand for higher safety and reliability standards in modern society. In generally, faults are unavoidable under practical conditions, such as hotspot faults, sensor faults and short circuits faults [1-3]. Up to now, various kinds of fault detection techniques have been developed, for example, model-based approaches, knowledge-based schemes and signal-based methods etc. Particularly, the problem of $H_{\infty}$ optimization-based fault detection has been an active research area [4-7]. However, in many practical cases, the fault detection systems have feedback control, that is, the fault detection system is usually of closed-loop type, and if the fault detection systems are designed separately from the control algorithms, faults may be hidden by control actions and the early detection of faults is clearly more difficult, especially low frequency faults [8-15]. Therefore, the problem of merging the control and fault detection units into a single detector/controller unit, i.e. simultaneous control and fault detection issue has become a very important research topic in information security field; recently. [8] dealt with the problem of simultaneous finitetime control and fault detection for linear switched systems with state delay and parameter

(c) The Author(s) 2018. This article is distributed under the terms of the Creative Commons Attribution 4.0 International License (http://creativecommons.org/licenses/by/4.0/), which permits unrestricted use, distribution, and reproduction in any medium, provided you give appropriate credit to the original author(s) and the source, provide a link to the Creative Commons license, and indicate if changes were made. 
uncertainties; [9] was concerned with the simultaneous robust control and fault detection problem for continuous-time switched systems subject to dwell time constraint; [10] investigated the problem of simultaneous fault detection and control for switched linear systems under a mixed $H_{\infty} / H_{-}$framework and [11] presented the problem of simultaneous fault detection and control design for switched systems with two quantized signals; in [12], the authors first attempted to deals with the simultaneous robust fault detection and control problem for a class of nonlinear stochastic switching systems under asynchronous switching; this paper further improved the results in the literature [9].

In practical situations, the periodical sampling is often used to control physical plants since it can simplify the design and analysis [16]. However, the communication burden is neglected in the framework of the periodic sampling; especially when the difference between consecutive sample-data is not distinct, it is obviously a waste of limited communication resources transmitting the sampled data to the controller [17]. Recently, in order to overcome this difficulty, event-triggered scheme is introduced and has been received particular attention, which is more convenient and effective than the traditional time-triggered technique, meantime, compared with the time-triggered mechanism, the event-triggered scheme can promise energy efficiency and reduce the burden of the communication [18-21].

In most existing literature, finite-time stability has received increasing attention and the concept of finite-time stability was proposed in practical processes, such as avoiding saturation or the excitation of nonlinear dynamics during the transient [22, 23]. Different from the classical Lyapunov stability concept, finite-time stability is defined as the behavior of the dynamical systems that can be tracked over a fixed finite-time interval, that is, the system state does not exceed a certain bound during a fixed finite-time interval. The introduction of such a stability concept is very necessary and important in many practical problems. So, the problem of finite-time stability it is not only the need of theoretical learning, but also the need of practical application. Now, many interesting results have been obtained for this type of stability. For example, [24] investigated the problem of robust finite-time boundedness of $H_{\infty}$ filtering for switch systems with time-varying delay; [25] addressed a finite-time stabilization problem for a class of continuous-time Markovian jump delay systems with switching control approach; [26] studied observerbased state feedback finite-time control for nonlinear jump systems with time-delay and [27] dealt with the finite-time synchronization problem for a class of uncertain coupled switched neural networks under asynchronous switching.

It is well known that singular systems, which are also referred to as generalized statespace systems, descriptor systems or implicit systems, as a kind of important system, singular systems have been received extensively attention during the past decade due basically to their powerful applications in many practical systems, such as economic systems, robotic systems, biological systems network control systems, chemical systems, and many other systems. Different from other regular systems, singular systems are more general and complex owing to not only the stability are need to be considered, but also regularity and absence of impulses or causality is need to be considered. Due to this fact singular systems can better describe and analyze the behavior of some physical systems than regular ones by standard state-space systems [28, 29]. Moreover, since the existence of time-delays often causes undesirable behavior such as degradation stability in dynamical systems, one of 
the major issues of stability and control analysis for time-delay singular systems has been studied extensively in actual problems [30,31].

The main challenge is now simultaneous finite-time control and fault detection in the presence of some complicated factors, such as jump model uncertainty, mixed delay and disturbances for a class of singular Markovian jump delay systems under asynchronous switching. To the best of our knowledge, this problem has not been investigated yet. Therefore, motivated by the aforementioned observations, in this paper, we will deal with the problem of simultaneous finite-time event-triggered control and fault detection for a class of singular Markovian systems with asynchronous switching signal, which based on some novel integral inequalities and average dwell time method. The purpose of this paper is to design mode-dependent detector/controller unit such that the augmented system is not only singular stochastic finite-time stability but also satisfies $H_{\infty}$ performance indices. A novel stochastic Lyapunov function and a set of strict LMIs will be utilized to derive sufficient conditions guaranteeing the desired detector/controller unit can be constructed. The main contributions of this paper can be summarized as follows: (1) In this paper, a class of much more general singular systems including both stochastic switch, deterministic switch and mixed time-varying delay are considered simultaneously. (2) Based on the event-triggered scheme, the simultaneous finite-time control and fault detection problem under asynchronous switching for a class of singular Markovian jump system is considered for the first time. (3) Compared with the method in [8], some novel sufficient conditions for singular stochastic finite-time stability of Markovian jump systems are obtained in this paper by virtue of new integral inequalities and asynchronous analysis method. (4) A mode-dependent controller/detector, which subject to the ADT constraint is designed, the elements of the transition rate matrix is modeled as a function of the high-level switching signal $\vartheta_{t}=p, \tilde{\vartheta}_{t}=q$; furthermore, owing to the bounded uncertainty description, the uncertainty entry in the transition rate matrix is represented by its upper and lower bounds.

Notations The notations are quite standard. Throughout this letter $\mathbb{R}^{n}$ and $\mathbb{R}^{n \times m}$ denote, respectively, the $\mathrm{n}$-dimensioned Euclidean space and the set of all $n \times m$ real matrices. The notation $X \geq Y$ (respectively, $X>Y$ ) means that $X$ and $Y$ are symmetric matrices, and that $X-Y$ is positive semi-definitive (respectively, positive definite). $L_{2}[0,+\infty$ ) is square integrable function vector over $[0,+\infty) \cdot\|\cdot\|$ is the Euclidean norm in $\mathbb{R}^{n}$. $I$ is the identity matrix with appropriate dimensions. $X+X^{T}$ is denoted $\mathrm{He}(X)$ for simplicity. If $A$ is a matrix, $\lambda_{\max }(A)$ (respectively, $\lambda_{\min }(A)$ ) means the largest (respectively, smallest) eigenvalue of $A$. Moreover, let $\left(\Omega, \mathscr{F},\left(\mathscr{F}_{t}\right)_{t \geq 0}, \mathbb{P}\right)$ be a complete probability space with a filteration. $\left(\mathbb{F}_{t}\right)_{t \geq 0}$ satisfies the usual conditions (i.e., the filtration contains all $P$-null sets and is right continuous). $\mathscr{E}\{\cdot\}$ stands for the mathematical expectation operator with respect to the given probability measure. Denote by $L_{\mathbb{F}_{0}}^{2}\left(\left[-d_{\tau}, 0\right]: \mathbb{R}^{n}\right)$ the family of all $\mathbb{F}_{0}$ measurable $C\left(\left[-d_{\tau}, 0\right]: \mathbb{R}^{n}\right)$-valued random variables $\varphi=\left\{\varphi(s):-d_{\tau} \leq s \leq 0\right\}$ such that $\sup _{-d_{\tau} \leq s \leq 0} \mathscr{E}\|\varphi(s)\|^{2}<\infty$. The asterisk $*$ in a matrix is used to denote a term that is induced by symmetry. Matrices, if not explicitly specified, are assumed to have appropriate dimensions. Sometimes, the arguments of the function will be omitted in the analysis when no confusion can arise. 


\section{Problem formulation and preliminaries}

Consider a class of SMJDSs described by the following model:

$$
\left\{\begin{aligned}
E\left(\vartheta_{t}\right) \dot{x}(t)= & A\left(v_{t}, \vartheta_{t}\right) x(t)+A_{d}\left(v_{t}, \vartheta_{t}\right) x(t-d(t))+A_{\tau}\left(v_{t}, \vartheta_{t}\right) \int_{t-\tau(t)}^{t} x(s) d s \\
& +B\left(v_{t}, \vartheta_{t}\right) u(t)+B_{h}\left(v_{t}, \vartheta_{t}\right) h(t)+B_{f}\left(v_{t}, \vartheta_{t}\right) f(t), \\
y(t)= & C_{1}\left(\vartheta_{t}\right) x(t)+D\left(\vartheta_{t}\right) x(t-d(t))+D_{\tau}\left(\vartheta_{t}\right) \int_{t-\tau(t)}^{t} x(s) d s+D_{h}\left(\vartheta_{t}\right) h(t) \\
& +D_{f}\left(\vartheta_{t}\right) f(t), \\
x(t)= & \phi(t), \quad t \in\left[-d_{\tau}, 0\right],
\end{aligned}\right.
$$

where $x(t) \in \mathbb{R}^{n}$ is the system state vector, $y(t) \in \mathbb{R}^{m}$ is the measured output, $h(t) \in \mathbb{R}^{l}$ is disturbance input, $u(t) \in \mathbb{R}^{g}$ is the control input and $f(t) \in \mathbb{R}^{v}$ is the fault vector. The matrix $E\left(\vartheta_{t}\right) \in \mathbb{R}^{n \times n}$ may be singular, and it is assumed that $\operatorname{rank}\left(E\left(\vartheta_{t}\right)\right)=r \leq n$. $\phi(t)$ is a vector-valued initial continuous function defined on the interval $\left[-d_{\tau}, 0\right] . \vartheta_{t}$ is a piecewise constant switching signal taking values in $\Delta_{1}=\{1,2, \ldots, \iota\}, \iota \in \mathbb{N}$. Suppose $t_{0}<t_{1}<\cdots$ is the switching sequence, then the system switches at instants $t_{0}<t_{1}<\cdots$, and $\vartheta(t)=\vartheta\left(t_{l}\right)$ for $\forall t \in\left[t_{l}, t_{l+1}\right)$, and $l=0,1, \ldots$.

Assumption 2.1 The input matrices $B\left(v_{t}, \vartheta_{t}\right)$ are full column rank and therefore, there exists nonsingular matrices $\bar{T}\left(v_{t}, \vartheta_{t}\right)$ such that $\bar{T}\left(v_{t}, \vartheta_{t}\right) B\left(v_{t}, \vartheta_{t}\right)=\left[\begin{array}{l}I \\ 0\end{array}\right]$.

Assumption 2.2 In this paper, the time-varying delays $d(t), \tau(t)$ are continuous satisfying $0 \leq d_{1} \leq d(t) \leq d_{2}, \dot{d}(t) \leq d<1$ and $0 \leq \tau_{1} \leq \tau(t) \leq \tau_{2}$, where $d_{1}, \tau_{1}$ and $d_{2}, \tau_{2}$ are constants involving the lower and the upper bounds of the delays, $d_{\tau}=\max \left\{d_{2}, \tau_{2}\right\}, \bar{d}=d_{2}-d_{1}, \bar{\tau}=$ $\tau_{2}-\tau_{1}$.

In this paper, the aim is to design a detector (filter)/controller (state feedback) unit, which is described by

$$
\left\{\begin{array}{l}
E\left(\tilde{\vartheta}_{t}\right) \dot{x}_{f}(t)=\hat{A}\left(v_{t}, \tilde{\vartheta}_{t}\right) x_{f}(t)+\hat{B}\left(v_{t}, \tilde{\vartheta}_{t}\right) y(t), \\
r(t)=\hat{C}_{r}\left(v_{t}, \tilde{\vartheta}_{t}\right) x_{f}(t)+\hat{D}_{r}\left(v_{t}, \tilde{\vartheta}_{t}\right) y(t), \\
u(t)=\hat{K}\left(v_{t}, \tilde{\vartheta}_{t}\right) x_{f}\left(\tilde{t}_{\sigma}\right), \quad t \in\left[\tilde{t}_{\sigma}, \tilde{t}_{\sigma+1}\right), \forall \sigma \in \mathbb{N},
\end{array}\right.
$$

where $x_{f}(t) \in \mathbb{R}^{n}$ is the state of the detector/controller unit, $r(t) \in \mathbb{R}^{l_{1}}$ is the residual signal, the matrices $\hat{A}\left(v_{t}, \tilde{\vartheta}_{t}\right), \hat{B}\left(v_{t}, \tilde{\vartheta}_{t}\right), \hat{C}_{r}\left(v_{t}, \tilde{\vartheta}_{t}\right), \hat{D}_{r}\left(v_{t}, \tilde{\vartheta}_{t}\right)$ and $\hat{K}\left(v_{t}, \tilde{\vartheta}_{t}\right)$ are detector/controller gains, which will be determined. Moreover, $\tilde{\vartheta}_{t}$ is the switching signal of detector/controller unit and can be regarded as a delayed signal of $\vartheta_{t}$. Under the switching signal $\vartheta_{t}$ and $\tilde{\vartheta}_{t}$, one can get the following switching sequences and Fig. 1 shows the phenomenon of asynchronous switching:

$$
\left\{\begin{array}{l}
\vartheta_{t}:\left\{\left(t_{0}, \vartheta\left(t_{0}\right)\right),\left(t_{1}, \vartheta\left(t_{1}\right)\right), \ldots,\left(t_{k}, \vartheta\left(t_{k}\right)\right), \ldots\right\}, \\
\tilde{\vartheta}_{t}:\left\{\left(t_{0}, \vartheta\left(t_{0}\right)\right),\left(t_{1}+d_{1}, \vartheta\left(t_{1}\right)\right), \ldots,\left(t_{k}+d_{k}, \vartheta\left(t_{k}\right)\right), \ldots\right\}
\end{array}\right.
$$

which indicates that if $t \in\left[t_{k}, t_{k}+d_{k}\right)$, then the $p$ th subsystem is coupled with the $q$ th detector/controller unit, and while $t \in\left[t_{k}+d_{k}, t_{k+1}\right)$, then the $p$ th subsystem is coupled with the $\mathrm{p}$ th detector/controller unit. Hence, $d_{k}$ represents the time lag of the switching 


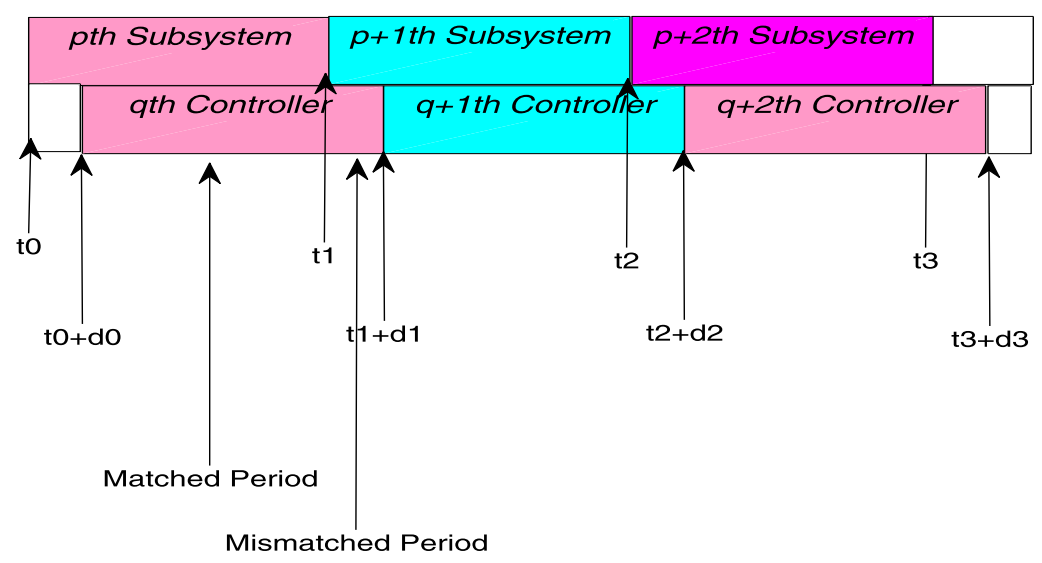

Figure 1 Asynchronous switching between subsystem and controller unit

instant of detector/controller unit to that of the system (1), and it is assumed that $\tilde{d}=$ $\max \left\{d_{k} \mid k=0,1, \ldots\right\}$ cannot exceed the next switching instant of the system.

$\left\{v_{t}, t \geq 0\right\}$ is a continuous-time discrete state Markov process with right continuous trajectory values in a finite set $\mathcal{K}=\{1,2, \ldots, \tilde{k}\}, \tilde{k} \in \mathbb{N}$ with the TPs

$$
\operatorname{Pr}\left(v_{t+\Delta}=j \mid v_{t}=i, \vartheta_{t}=p, \tilde{\vartheta}_{t}=q\right)= \begin{cases}\pi_{i j}^{(p q)} \Delta+o(\Delta), & i \neq j, \\ 1+\pi_{i i}^{(p q)} \Delta+o(\Delta), & i=j,\end{cases}
$$

where $\Delta>0, \lim _{\Delta \rightarrow 0^{+}} o(\Delta) / \Delta=0$ and $\pi_{i j}^{(p q)}$ is the transition rate from mode $i$ at time $t$ to mode $j$ at time $t+\Delta$ that satisfies $\pi_{i j}^{(p q)}>0, \pi_{i i}^{(p q)}=-\sum_{j=1, j \neq i}^{\tilde{k}} \pi_{i j}^{(p q)}\left(\forall i, j \in \mathcal{K}, \forall p, q \in \Delta_{1}\right)$.

Remark 2.1 Based on the definition of the transition rate matrix $\Pi^{(p q)}$, we know that every element $\pi_{i j}^{(p q)}$ of the matrix $\Pi^{(p q)}$ is a function of the switch mode $\vartheta_{t}=p, \tilde{\vartheta}_{t}=q$, it means that this matrix can be defined as

$$
\Pi^{(p q)}=\left[\begin{array}{ccc}
\pi_{11}^{(p q)} & \cdots & \pi_{1 \tilde{k}}^{(p q)} \\
\vdots & \ddots & \vdots \\
\pi_{\tilde{k} 1}^{(p q)} & \cdots & \pi_{\tilde{k} \tilde{k}}^{(p q)}
\end{array}\right] .
$$

Note that matrix $\Pi^{(p q)}$ is the time-varying and subject to the ADT constraint. Such timevarying transition rates $\pi_{i j}^{(p q)}$ are unknown but they belong to the admissible bounded compact set

$$
\pi_{i j}^{(p q)}=\bar{\pi}_{i j}^{(p q)}+\Delta \bar{\pi}_{i j}^{(p q)}
$$

and the uncertain $\Delta \bar{\pi}_{i j}^{(p q)}$ belongs to the range of $\left[-\delta_{i j}^{(p q)}, \delta_{i j}^{(p q)}\right]$, where $\delta_{i j}^{(p q)}>0$, for all $i, j \in$ $\mathcal{K}, p, q \in \Delta_{1}$. The entry of $\bar{\pi}_{i j}^{(p q)}$ and $\delta_{i j}^{(p q)}$ satisfy $\bar{\pi}_{i i}^{(p q)}=-\sum_{\substack{j=1 \\ j \neq i}}^{\tilde{k}} \bar{\pi}_{i j}^{(p q)}, \delta_{i i}^{(p q)}=\sum_{\substack{j=1 \\ j \neq i}}^{\tilde{k}} \delta_{i j}^{(p q)}$.

Now, we will introduce the following event-trigger instant sequence:

$$
\chi=\left\{\left[\tilde{t}_{0}, \tilde{t}_{1}\right),\left[\tilde{t}_{1}, \tilde{t}_{2}\right), \ldots,\left[\tilde{t}_{\sigma}, \tilde{t}_{\sigma+1}\right), \ldots \mid \sigma \in \mathbb{N}\right\} .
$$


Without loss of generality, we assume that the first event happens at time instant $\tilde{t}_{0}$. With the system state estimates $x_{f}\left(\tilde{t}_{\sigma}\right)$ sampled at time instant $\tilde{t}_{\sigma}$, the next sampling instant $\tilde{t}_{\sigma+1}$ can be determined by the event-trigger. In this paper, the state feedback controller is event-triggered, which can be given by

$$
u(t)=\hat{K}\left(v_{t}, \tilde{\vartheta}_{t}\right) x_{f}\left(\tilde{t}_{\sigma}\right), \quad t \in\left[\tilde{t}_{\sigma}, \tilde{t}_{\sigma+1}\right), \forall \sigma \in \mathbb{N} .
$$

Note that in the event-triggered scenario, at sampling time instant $\tilde{t}_{\sigma}$, the controller (8) will receive the sampled data and hold unchanged until next event is generated at time instant $\tilde{t}_{\sigma+1}$, that is, there is no new control input updates during two consecutive time instants in sequence $\chi$. Hence, a zero-order holder $(\mathrm{ZOH})$ is equipped in the system in order to keep the control signal continuous.

Now, we introduce the event detector which is used to determined whether the newly sampled data should be sent out to the controller by using the following threshold condition:

$$
e^{T}(t) \Phi_{1 i} e(t)-\rho_{i} x_{f}^{T}(t) \Phi_{2 i} x_{f}(t) \leq 0,
$$

where $e(t)=x_{f}\left(\tilde{t}_{\sigma}\right)-x_{f}(t), \Phi_{1 i}>0$ and $\Phi_{2 i}>0$ for each $i \in \mathcal{K}$, are an event-triggered weighting matrix to be determined for a proper error tolerance $\rho_{i} \in[0,1)$. Based on the above inequality, we know that if the sampling data exceeds the threshold condition (9), the $(\sigma+1)$ th event will be triggered.

Remark 2.2 Based on (9), we know that the next event will not be generated before $e^{T}(t) \Phi_{1 i} e(t)-\rho_{i} x_{f}^{T}(t) \Phi_{2 i} x_{f}(t)=0$ and at the event-trigger instant $\tilde{t}_{\delta}$, if $e(t)=0$, one sees that a positive lower bound exists on the inter-event, that is, $\tilde{t}_{\delta+1}-\tilde{t}_{\delta}>0$, which eliminates the Zeno behavior of the sampling. Furthermore, by the similar method, which was discussed in [18], a positive lower bound will be obtained. Therefore, based on the above description, the Zeno behavior of the sampling can be excluded because there is no accumulation point in the sampling if

$$
\tilde{t}_{\delta+1}=\inf \left\{t>\tilde{t}_{\delta} \mid e^{T}(t) \Phi_{1 i} e(t)-\rho_{i} x_{f}^{T}(t) \Phi_{2 i} x_{f}(t) \geq 0\right\} \quad \forall \delta \in \mathbb{N}
$$

holds.

Then, by combining the detector/controller unit (2) and the system (1), we can obtain the following augmented system:

$$
\left\{\begin{aligned}
\tilde{E}_{p q} \dot{\tilde{x}}(t)= & \tilde{A}_{i p q} \tilde{x}(t)+\tilde{A}_{\text {dipq }} \tilde{x}(t-d(t)) \\
& +\tilde{A}_{\tau i p q} \int_{t-\tau(t)}^{t} \tilde{x}(s) d s+\tilde{A}_{e i p q} e(t)+\tilde{B}_{z i p q} z(t), \\
\hat{r}(t)= & \bar{C}_{r i p q} \tilde{x}(t)+\hat{D}_{r i q} D_{p} k \tilde{x}(t-d(t)) \\
& +\hat{D}_{r i q} D_{\tau p} k \int_{t-\tau(t)}^{t} \tilde{x}(s) d s+\bar{D}_{r i p q} z(t)-C_{w} x_{w}(t) .
\end{aligned}\right.
$$

Case I: switching signals $\vartheta_{t}$ and $\tilde{\vartheta}_{t}$ are a mismatch, then

$$
\tilde{E}_{p q}=\left[\begin{array}{cc}
E_{p} & 0 \\
0 & E_{q}
\end{array}\right], \quad \tilde{A}_{i p q}=\left[\begin{array}{cc}
A_{i p} & B_{p} \hat{K}_{i q} \\
\hat{B}_{i q} C_{1 p} & \hat{A}_{i q}
\end{array}\right], \quad \tilde{A}_{\text {dipq }}=\left[\begin{array}{cc}
A_{\text {dip }} & 0 \\
\hat{B}_{i q} D_{p} & 0
\end{array}\right],
$$




$$
\begin{aligned}
& \tilde{A}_{\tau i p q}=\left[\begin{array}{c}
A_{\tau i p} k \\
\hat{B}_{i q} D_{\tau p} k
\end{array}\right], \quad \tilde{A}_{\text {eipq }}=\left[\begin{array}{c}
B_{i p} \hat{K}_{i q} \\
0
\end{array}\right], \quad \tilde{B}_{z i p q}=\left[\begin{array}{cc}
B_{h i p} & B_{f i p} \\
\hat{B}_{i q} D_{h p} & \hat{B}_{i q} D_{f p}
\end{array}\right], \\
& \bar{D}_{\text {ripq }}=\left[\begin{array}{ll}
\hat{D}_{r i q} D_{h p} & \hat{D}_{r i q} D_{f p}-D_{w}
\end{array}\right], \quad \bar{C}_{r i p q}=\left[\begin{array}{ll}
\hat{D}_{r i q} C_{1 p} & \hat{C}_{r i q}
\end{array}\right], \\
& \tilde{x}(t)=\left[\begin{array}{c}
x(t) \\
x_{f}(t)
\end{array}\right], \quad \hat{r}(t)=r(t)-r_{w}(t), \quad\left[\frac{k}{k^{\prime}}\right]=\left[\begin{array}{ll}
I & 0 \\
\hline 0 & I
\end{array}\right], \quad z(t)=\left[\begin{array}{l}
h(t) \\
f(t)
\end{array}\right] .
\end{aligned}
$$

Furthermore, $\hat{r}(t)$ and $r_{w}(s)=W(s) f(s)$ are a filtered version of the fault signals and $W(s) \in$ $R H_{\infty}$ is the given stable weighting transfer function. The realization of $r_{w}(s)=W(s) f(s)$ is supposed to be

$$
W:\left\{\begin{array}{l}
\dot{x}_{w}(t)=A_{w} x_{w}(t)+B_{w} f(t), \\
r_{w}(t)=C_{w} x_{w}(t)+D_{w} f(t), \\
x_{w}(0)=0
\end{array}\right.
$$

where $A_{w}, B_{w}, C_{w}$, and $D_{w}$ are known constant matrices.

Case II: switching signals $\vartheta_{t}$ and $\tilde{\vartheta}_{t}$ are matched, then

$$
\begin{aligned}
& \tilde{E}_{p q}=\tilde{E}_{p}=\left[\begin{array}{cc}
E_{p} & 0 \\
0 & E_{p}
\end{array}\right], \quad \tilde{A}_{i p q}=\tilde{A}_{i p}=\left[\begin{array}{cc}
A_{i p} & B_{p} \hat{K}_{i p} \\
\hat{B}_{i q} C_{1 p} & \hat{A}_{i p}
\end{array}\right], \\
& \tilde{A}_{\text {dipq }}=\tilde{A}_{\text {dip }}=\left[\begin{array}{cc}
A_{\text {dip }} & 0 \\
\hat{B}_{i p} D_{p} & 0
\end{array}\right], \quad \tilde{A}_{\tau i p q}=\tilde{A}_{\tau i p}=\left[\begin{array}{c}
A_{\tau i p} k \\
\hat{B}_{i p} D_{\tau p} k
\end{array}\right], \\
& \tilde{A}_{\text {eipq }}=\tilde{A}_{\text {eip }}=\left[\begin{array}{c}
B_{i p} \hat{K}_{i p} \\
0
\end{array}\right], \quad \tilde{B}_{z i p q}=\tilde{B}_{z i p}=\left[\begin{array}{cc}
B_{h i p} & B_{f i p} \\
\hat{B}_{i p} D_{h p} & \hat{B}_{i p} D_{f p}
\end{array}\right], \\
& \bar{D}_{\text {ripq }}=\bar{D}_{\text {rip }}=\left[\begin{array}{ll}
\hat{D}_{\text {rip }} D_{h p} & \hat{D}_{r i p} D_{f p}-D_{w}
\end{array}\right], \quad \bar{C}_{\text {ripq }}=\bar{C}_{r p}=\left[\begin{array}{ll}
\hat{D}_{r i p} C_{1 p} & \hat{C}_{r i p}
\end{array}\right] .
\end{aligned}
$$

Remark 2.3 In this paper, the simultaneous finite-time control and fault detection problem will be described as designing a detector/controller unit in form of (2) such that the augmented system (10) is SSFTS and the following $H_{\infty}$ property should be guaranteed when there exists a disturbance and fault under zero initial conditions:

$$
\mathscr{E}\left\{\int_{0}^{T} e^{-\lambda s} \hat{r}^{T}(s) \hat{r}(s) d s\right\} \leq \gamma^{2} \mathscr{E}\left\{\int_{0}^{T} z^{T}(s) z(s) d s\right\}, \quad \lambda, \gamma>0
$$

Remark 2.4 Recently, $[18,19]$ investigated the problem of fault detection for networked control systems based on event-triggering mechanism, however, the fault detection filter is designed separately from the state feedback control algorithms. Furthermore, in [20], the authors continued to study the problem of event-triggered fault detection filter and controller coordinated design for networked control system, obviously, the results are much more general than the conclusions of $[18,19]$. In [12], the authors discussed the problem of fault detection and control for stochastic switched delay systems under asynchronous conditions for the first time. In summary, to the best of our knowledge, the same problem about singular MJDSs using an event-triggered sampling scheme has not been investi- 
gated yet, and the results show great room to improve by introducing some novel integral inequalities. So, this is the main motivation for us to further develop the simultaneous finite-time control and fault detection problem.

Now, the following definitions are given, which are indispensable for later development.

Definition 2.1 ([32]) For any switching signal and any $k_{0}<k_{s}<k$, let $N_{\delta}\left(k_{s}, k\right)$ denote the number of switching signals over the time interval $\left[k_{s}, k\right)$. For given $N_{0}>0, \tau_{a}>0$, then

$$
N_{\delta}\left(k_{s}, k\right) \leq N_{0}+\frac{k-k_{s}}{\tau_{a}},
$$

where $\tau_{a}$ is called average dwell time and $N_{0}$ denotes the chatter bound.

Definition $2.2([33])$ system $\tilde{E}_{p q} \dot{\tilde{x}}(t)=\tilde{A}_{i p q} \tilde{x}(t)$ (or pair $\left(\tilde{E}_{p q}, \tilde{A}_{i p q}\right)$ ) is said to be:

1. regular if $\operatorname{det}\left(z \tilde{E}_{p q}-\tilde{A}_{i p q}\right)$ is not identically zero for any $i \in \mathcal{K}, p, q \in \Delta_{1}$;

2. impulse-free if it is regular and $\operatorname{degree}\left(\operatorname{det}\left(z \tilde{E}_{p q}-\tilde{A}_{i p q}\right)\right)=\operatorname{rank}\left(\tilde{E}_{p q}\right)$ for any $i \in \mathcal{K}$,

$p, q \in \Delta_{1}$.

Definition 2.3 ([34]) The augmented system (10) is said to be SSFTS with respect to $\left(c_{1}, c_{2}, \mathscr{G}, T\right)$. With $0<c_{1}<c_{2}, \mathscr{G}>0$, if the stochastic system is regular and impulse-free in time $t \in\left[t_{0}, T\right]$ and satisfies

$$
\begin{aligned}
& \sup _{t_{0}-d_{\tau} \leq t \leq 0} \mathscr{E}\left\{\tilde{x}^{T}(t) \mathscr{G} \tilde{x}(t), x_{w}^{T}(t) \mathscr{G}_{x_{w}}(t), \dot{\tilde{x}}^{T}(t) \mathscr{G} \dot{\tilde{x}}(t)\right\} \leq c_{1} \\
& \quad \Rightarrow \quad \mathscr{E}\left\{\tilde{x}^{T}(t) \mathscr{G} \tilde{x}(t)\right\}<c_{2}, \quad t \in\left[t_{0}, T\right] .
\end{aligned}
$$

Before proceeding, we will introduce the following lemmas which will play an important role in the derivation of our main results.

Lemma 2.1 ([35]) For a differentiable function $x:[\alpha, \beta] \rightarrow \mathbb{R}^{n}$, a positive definite matrix $R \in \mathbb{R}^{n \times n}$, a vector $\xi \in \mathbb{R}^{k}$, and any matrices $N_{i} \in \mathbb{R}^{n \times n}(i=1,2)$, the following inequality hold:

$$
\begin{aligned}
& -\int_{\alpha}^{\beta} \dot{x}^{T}(s) R \dot{x}(s) d s \\
& \quad \leq \xi^{T}\left[(\alpha-\beta)\left(N_{1} R^{-1} N_{1}^{T}+\frac{1}{3} N_{2} R^{-1} N_{2}^{T}\right)+H e\left(N_{1} E_{1}+N_{2} E_{2}\right)\right] \xi,
\end{aligned}
$$

where

$$
\begin{aligned}
& E_{1} \xi=\Upsilon_{1}(\alpha, \beta)=x(\beta)-x(\alpha), \\
& E_{2} \xi=\Upsilon_{2}(\alpha, \beta)=x(\beta)+x(\alpha)-\frac{2}{\beta-\alpha} \int_{\alpha}^{\beta} x(s) d s .
\end{aligned}
$$


Lemma 2.2 For any appropriately dimensioned matrices $Z=Z^{T}>0, Z \in \mathbb{R}^{n \times n}, M \in \mathbb{R}^{m \times n}$ and positive scalars $d_{1}$, and $\alpha$, the following inequality holds:

$$
\begin{aligned}
& -\int_{t-d_{1}}^{t} e^{\alpha_{2}(t-s)} \dot{\tilde{x}}^{T}(s) \tilde{E}^{T} Z \tilde{E} \dot{\tilde{x}}(s) d s \\
& \quad \leq \xi^{T}(t) \varepsilon M Z^{-1} M^{T} \xi(t)+2 \xi^{T}(t) M\left(\tilde{E} \tilde{x}(t)-\tilde{E} \tilde{x}\left(t-d_{1}\right)\right),
\end{aligned}
$$

where $\varepsilon=\frac{1}{\alpha_{2}}\left(1-e^{-\alpha_{2} d_{1}}\right)$.

Proof Firstly, we can find that the following inequality is true

$$
\int_{t-d_{1}}^{t}\left[\begin{array}{c}
\xi(t) \\
\tilde{E} \tilde{\tilde{x}}(s)
\end{array}\right]^{T}\left[\begin{array}{c}
e^{-\frac{\alpha(t-s)}{2}} M \\
e^{\frac{\alpha(t-s)}{2}} Z
\end{array}\right] Z^{-1}\left[e^{-\frac{\alpha(t-s)}{2}} M^{T} \quad e^{\frac{\alpha(t-s)}{2}} Z\right]\left[\begin{array}{c}
\xi(t) \\
\tilde{E} \dot{\tilde{x}}(s)
\end{array}\right] d s \geq 0,
$$

which implies the inequality (16) is satisfied.

Remark 2.5 Lemma 2.1 and Lemma 2.2 represent some novel results to handle the integral term of quadratic quantities in the estimation of LKF derivative. Lemma 2.1 includes more information of state and time-varying delay into the augmented vectors $\xi(t)$; Lemma 2.2 contains the exponential information, and it does not use the approximation $-e^{\alpha(t-s)}<-1$, $t-d_{2} \leq s \leq t-d_{1}$, which presents less precision. So, Lemma 2.1 and Lemma 2.2 are helpful to reduce the imprecision of our results.

Lemma 2.3 ([36]) For any constant matrix $M>0$, any scalars $a$ and $b$ with $a<b$, and $a$ vector function $x(t):[a, b] \rightarrow \mathbb{R}^{n}$ such that the integrals concerned are well defined, then the following inequality holds:

$$
\left[\int_{a}^{b} x(s) d s\right]^{T} M\left[\int_{a}^{b} x(s) d s\right] \leq(b-a) \int_{a}^{b} x^{T}(s) M x(s) d s
$$

\section{Design of the detector/controller unit}

In this section, LMI conditions are presented such that the SSFTS and performance property (12) are satisfied simultaneously for augmented system (10) with average dwell time constraint and then we will endeavour to develop the design problems for detector/controller unit with the form of (2).

Theorem 3.1 For any $i, j \in \mathcal{K}, p, q \in \Delta_{1}, \alpha_{1}>0, \alpha_{2}>0, \gamma_{0}>0$, and given matrices $\hat{A}_{i q}$, $\hat{B}_{i q}, \hat{C}_{r i q}, \hat{D}_{r i q}, \hat{K}_{i q}, \tilde{R}_{p q}$, then the augmented system (10) with $z(t)=0$ is SSFTS with respect given $\left(c_{1}, c_{2}, \mathscr{G}, T\right)$ and the performance index (12) is satisfied, if there exist positive definite matrices $P_{i p q}, P_{w p q}, Q_{l p q}, l=1,2,3, \bar{R}_{1 p q}, \bar{R}_{2 p q}, W_{1 p q}, \bar{W}_{1 p q}, W_{21 p q}, W_{22 p q}$ symmetric matrices $\mathscr{H}_{1}, \mathscr{H}_{2}$ and any real matrices $T_{k}, k=1,2,3,4,5, \mathfrak{G}, S_{p q}, S_{\text {wpq }}$, such that the following LMIs 
hold:

$$
\begin{aligned}
& \Theta_{i p q}^{d_{1}}=\left[\begin{array}{ccccccccc}
\Theta_{11}^{(p q)} & \Theta_{12}^{(p q)} & \Theta_{13}^{(p q)} & 0 & \Theta_{15}^{(p q)} & 0 & \Theta_{17}^{(p q)} & \Theta_{18}^{(p q)} & \Theta_{19}^{(p q)} \\
* & \Theta_{22}^{(p q)} & \Theta_{23}^{(p q)} & \Theta_{24}^{(p q)} & 0 & 0 & \Theta_{27}^{(p q)} & \Theta_{28}^{(p q)} & 0 \\
* & * & \Theta_{33}^{(p q)} & \Theta_{34}^{(p q)} & 0 & 0 & \Theta_{37}^{(p q)} & \Theta_{38}^{(p q)} & 0 \\
* & * & * & \Theta_{44}^{(p q)} & 0 & 0 & \Theta_{47}^{(p q)} & \Theta_{48}^{(p q)} & 0 \\
* & * & * & * & \Theta_{55}^{(p q)} & 0 & 0 & \Theta_{58}^{(p q)} & 0 \\
* & * & * & * & * & \Theta_{66}^{(p q)} & \Theta_{67}^{(p q)} & \Theta_{68} & 0 \\
* & * & * & * & * & * & \Theta_{77}^{(p q)} & \Theta_{78}^{(p q)} & 0 \\
* & * & * & * & * & * & * & \Theta_{88}^{(p q)} & 0 \\
* & * & * & * & * & * & * & * & \Theta_{99}
\end{array}\right]<0 \\
& \Theta_{i p q}^{d_{2}}=\left[\begin{array}{ccccccccc}
\Theta_{11}^{(p q)} & \Theta_{12}^{(p q)} & \Theta_{13}^{(p q)} & 0 & \Theta_{15}^{(p q)} & 0 & \Theta_{17}^{(p q)} & \Theta_{18}^{(p q)} & \Theta_{19}^{(p q)} \\
* & \Theta_{22}^{(p q)} & \Theta_{23}^{(p q)} & \Theta_{24}^{(p q)} & 0 & 0 & \Theta_{27}^{(p q)} & \bar{\Theta}_{28}^{(p q)} & 0 \\
* & * & \Theta_{33}^{(p q)} & \Theta_{34}^{(p q)} & 0 & 0 & \Theta_{37}^{(p q)} & \bar{\Theta}_{38}^{(p q)} & 0 \\
* & * & * & \Theta_{44}^{(p q)} & 0 & 0 & \Theta_{47}^{(p q)} & 0 & 0 \\
* & * & * & * & \Theta_{55}^{(p q)} & 0 & 0 & \Theta_{58}^{(p q)} & 0 \\
* & * & * & * & * & \Theta_{66}^{(p q)} & \Theta_{67}^{(p q)} & \Theta_{68} & 0 \\
* & * & * & * & * & * & \Theta_{77}^{(p q)} & \bar{\Theta}_{78}^{(p q)} & 0 \\
* & * & * & * & * & * & * & \Theta_{88}^{(p q)} & 0 \\
* & * & * & * & * & * & * & * & \Theta_{99}
\end{array}\right]<0 \\
& \Theta_{i p}^{d_{1}}=\left[\begin{array}{ccccccccc}
\Theta_{11}^{(p)} & \Theta_{12}^{(p)} & \Theta_{13}^{(p)} & 0 & \Theta_{15}^{(p)} & 0 & \Theta_{17}^{(p)} & \Theta_{18}^{(p)} & \Theta_{19}^{(p)} \\
* & \Theta_{22}^{(p)} & \Theta_{23}^{(p)} & \Theta_{24}^{(p)} & 0 & 0 & \Theta_{27}^{(p)} & \Theta_{28}^{(p)} & 0 \\
* & * & \Theta_{33}^{(p)} & \Theta_{34}^{(p)} & 0 & 0 & \Theta_{37}^{(p)} & \Theta_{38}^{(p)} & 0 \\
* & * & * & \Theta_{44}^{(p)} & 0 & 0 & \Theta_{47}^{(p)} & \Theta_{48}^{(p)} & 0 \\
* & * & * & * & \Theta_{55}^{(p)} & 0 & 0 & \Theta_{58}^{(p)} & 0 \\
* & * & * & * & * & \Theta_{66}^{(p)} & \Theta_{67}^{(p)} & \Theta_{68} & 0 \\
* & * & * & * & * & * & \Theta_{77}^{(p)} & \Theta_{78}^{(p)} & 0 \\
* & * & * & * & * & * & * & \Theta_{88}^{(p)} & 0 \\
* & * & * & * & * & * & * & * & \Theta_{99}
\end{array}\right]<0, \\
& \Theta_{i p}^{d_{2}}=\left[\begin{array}{ccccccccc}
\Theta_{11}^{(p)} & \Theta_{12}^{(p)} & \Theta_{13}^{(p)} & 0 & \Theta_{15}^{(p)} & 0 & \Theta_{17}^{(p)} & \Theta_{18}^{(p)} & \Theta_{19}^{(p)} \\
* & \Theta_{22}^{(p)} & \Theta_{23}^{(p)} & \Theta_{24}^{(p)} & 0 & 0 & \Theta_{27}^{(p)} & \bar{\Theta}_{28}^{(p)} & 0 \\
* & * & \Theta_{33}^{(p)} & \Theta_{34}^{(p)} & 0 & 0 & \Theta_{37}^{(p)} & \bar{\Theta}_{38}^{(p)} & 0 \\
* & * & * & \Theta_{44}^{(p)} & 0 & 0 & \Theta_{47}^{(p)} & 0 & 0 \\
* & * & * & * & \Theta_{55}^{(p)} & 0 & 0 & \Theta_{58}^{(p)} & 0 \\
* & * & * & * & * & \Theta_{66}^{(p)} & \Theta_{67}^{(p)} & \Theta_{68} & 0 \\
* & * & * & * & * & * & \Theta_{77}^{(p)} & \bar{\Theta}_{78}^{(p)} & 0 \\
* & * & * & * & * & * & * & \Theta_{88}^{(p)} & 0 \\
* & * & * & * & * & * & * & * & \Theta_{99}
\end{array}\right]<0,
\end{aligned}
$$




$$
\begin{aligned}
& {\left[\begin{array}{cc}
W_{21 p q} & \mathscr{H}_{1} \\
\mathscr{H}_{1} & W_{22 p q}
\end{array}\right]>0, \quad\left[\begin{array}{cc}
W_{21 p q} & \mathscr{H}_{2} \\
\mathscr{H}_{2} & W_{22 p q}
\end{array}\right]>0,} \\
& {\left[\begin{array}{cc}
{\left[\begin{array}{cc}
W_{21 p q} & \mathscr{H}_{1} \\
\mathscr{H}_{1} & W_{22 p q}
\end{array}\right]} & \mathfrak{G} \\
\mathfrak{G}^{T} & {\left[\begin{array}{cc}
W_{21 p q} & \mathscr{H}_{2} \\
\mathscr{H}_{2} & W_{22 p q}
\end{array}\right]}
\end{array}\right]>0,} \\
& \eta_{1} c_{2} e^{-\alpha_{1} T_{\mathrm{m}}\left(t_{0}, T\right)-\alpha_{2} T_{\mathrm{mism}}\left(t_{0}, T\right)}>\eta_{2} c_{1} \text {, }
\end{aligned}
$$

and the average dwell time satisfying

$$
\begin{aligned}
\tau_{a}> & \tau_{a}^{*} \\
= & \max \left\{\frac{\left(\ln \left(\mu_{1} \mu_{2}\right)+\left|\alpha_{1}-\alpha_{2}\right| d_{\tau}\right) T}{\ln \frac{c_{2}}{c_{1}}+\ln \frac{\eta_{1}}{\eta_{2}}-\alpha_{1} T_{\mathrm{m}}\left(t_{0}, T\right)-\alpha_{2} T_{\mathrm{mism}}\left(t_{0}, T\right)},\right. \\
& \left.\frac{\ln \left(\mu_{1} \mu_{2}\right)+\left|\alpha_{1}-\alpha_{2}\right| d_{\tau}+\alpha_{2} \tilde{d}}{\alpha_{1}}\right\},
\end{aligned}
$$

where

$$
\begin{aligned}
& \Theta_{11}^{(p q)}=H e\left(\mathscr{M}_{i p q} \tilde{A}_{i p q}\right)-\alpha_{2} \tilde{E}_{p q}^{T} P_{i p q} \tilde{E}_{p q}+\sum_{v=1}^{3} Q_{v p q}+\tau_{1}^{2} \bar{R}_{1 p q} \\
& +\bar{\tau}^{2} \bar{R}_{2 p q}+H e\left(T_{51} \tilde{E}_{p q}\right)+\rho_{i} k^{\prime T} \Phi_{2 i} k^{\prime}+\bar{d}^{2} \tilde{E}_{p q}^{T} W_{21 p q} \tilde{E}_{p q} \\
& +\sum_{\substack{j=1 \\
j \neq i}}^{\tilde{k}} \bar{\pi}_{i j}^{(p q)} \tilde{E}_{p q}^{T}\left(P_{j p q}-P_{i p q}\right) \tilde{E}_{p q}+\sum_{\substack{j=1 \\
j \neq i}}^{\tilde{k}} \frac{1}{4}\left(\delta_{i j}^{(p q)}\right)^{2} \varepsilon_{i j}, \\
& \Theta_{12}^{(p q)}=\mathscr{M}_{i p q} \tilde{A}_{\text {dipq }}, \quad \Theta_{13}^{(p q)}=-T_{51} \tilde{E}_{p q}+\tilde{E}_{p q}^{T} T_{53}^{T}, \quad \Theta_{15}^{(p q)}=\left[\begin{array}{lll}
0 & \mathscr{M}_{i p q} \tilde{A}_{\text {cipq }} & 0
\end{array}\right], \\
& \Theta_{17}^{(p q)}=\left[\begin{array}{llll}
0 & 0 & \mathscr{M}_{i p q} \tilde{A}_{\text {eipq }} & \mathscr{M}_{i p q} \tilde{B}_{z i p q}
\end{array}\right], \quad \Theta_{18}^{(p q)}=\left[\begin{array}{llll}
\bar{C}_{r i p q}^{T} & \tilde{A}_{i p q}^{T} & 0_{n \times 2 n} & T_{51}
\end{array}\right], \\
& \Theta_{19}^{(p q)}=\left[\begin{array}{llll}
\tilde{E}_{p q}^{T}\left(P_{1 p q}-P_{i p q}\right) \tilde{E}_{p q} & \cdots & \tilde{E}_{p q}^{T}\left(P_{(i-1) p q}-P_{i p q}\right) \tilde{E}_{p q} & \tilde{E}_{p q}^{T}\left(P_{(i+1) p q}-P_{i p q}\right) \tilde{E}_{p q}
\end{array}\right. \\
& \left.\cdots \quad \tilde{E}_{p q}^{T}\left(P_{\tilde{k} p q}-P_{i p q}\right) \tilde{E}_{p q}\right] \text {, } \\
& \Theta_{22}^{(p q)}=-(1-d) e^{-\alpha_{2} d_{2}} Q_{1 p q}+H e\left(T_{12} \tilde{E}_{p q}+T_{22} \tilde{E}_{p q}-T_{32} \tilde{E}_{p q}+T_{42} \tilde{E}_{p q}-\tilde{E}_{p q}^{T} \mathfrak{G}_{2} \tilde{E}_{p q}\right) \\
& +\tilde{E}_{p q}^{T}\left(\bar{d}_{1} \mathscr{H}_{1}-\bar{d} \mathscr{H}_{2}\right) \tilde{E}_{p q}+2 \tilde{E}_{p q}^{T} W_{22 p q} \tilde{E}_{p q}, \\
& \Theta_{23}^{(p q)}=T_{32} \tilde{E}_{p q}-\tilde{E}_{p q}^{T} T_{33}^{T}+T_{42} \tilde{E}_{p q}+\tilde{E}_{p q}^{T} T_{43}^{T}+\tilde{E}_{p q}^{T} \mathfrak{G}_{2} \tilde{E}_{p q}-\tilde{E}_{p q}^{T} W_{22 p q} \tilde{E}_{p q}, \\
& \Theta_{24}^{(p q)}=-T_{12} \tilde{E}_{p q}+\tilde{E}_{p q}^{T} T_{14}^{T}+T_{22} \tilde{E}_{p q}+\tilde{E}_{p q}^{T} T_{24}^{T}+\tilde{E}_{p q}^{T} \mathfrak{G}_{2}^{T} \tilde{E}_{p q}-\tilde{E}_{p q}^{T} W_{22 p q} \tilde{E}_{p q},
\end{aligned}
$$

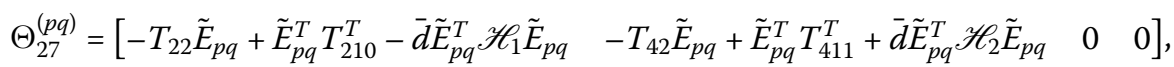

$$
\begin{aligned}
& \Theta_{28}^{(p q)}=\left[\begin{array}{lllll}
\left(\hat{D}_{\text {riq }} D_{p} k\right)^{T} & \tilde{A}_{\text {dipq }}^{T} & \sqrt{\bar{d}} T_{12} & \sqrt{\bar{d}} T_{22} & 0
\end{array}\right], \\
& \Theta_{33}^{(p q)}=-e^{\alpha_{2} d_{1}} Q_{2 p q}+H e\left(T_{33} \tilde{E}_{p q}+T_{43} \tilde{E}_{p q}-T_{53} \tilde{E}_{p q}\right)+\tilde{E}_{p q}^{T}\left(\bar{d} \mathscr{H}_{2}+W_{22 p q}\right) \tilde{E}_{p q} \Theta_{34}^{(p q)} \\
& =-\tilde{E}_{p q}^{T} G_{2}^{T} \tilde{E}_{p q} \\
& \Theta_{37}^{(p q)}=\left[\begin{array}{lll}
0 & -T_{43} \tilde{E}_{p q}+\tilde{E}_{p q}^{T} T_{411}^{T}-\bar{d} \tilde{E}_{p q}^{T} \mathscr{H}_{2} \tilde{E}_{p q} \quad 0 \quad 0
\end{array}\right], \quad \Theta_{38}^{(p q)}=\left[\begin{array}{ll}
0_{n \times 4 n} & T_{53}
\end{array}\right],
\end{aligned}
$$




$$
\begin{aligned}
& \Theta_{44}^{(p q)}=-e^{\alpha_{2} d_{2}} Q_{3 p q}+H e\left(-T_{14} \tilde{E}_{p q}+T_{24} \tilde{E}_{p q}\right)-\tilde{E}_{p q}^{T}\left(\bar{d} \mathscr{H}_{1}-W_{22 p q}\right) \tilde{E}_{p q}, \\
& \Theta_{47}^{(p q)}=\left[-2 T_{24} \tilde{E}_{p q}+\tilde{E}_{p q}^{T} T_{210}^{T}+\bar{d} \tilde{E}_{p q}^{T} \mathscr{H}_{1} \tilde{E}_{p q} \quad 0 \quad 0 \quad 0\right], \\
& \Theta_{48}^{(p q)}=\left[\begin{array}{lllll}
0 & 0 & \sqrt{\bar{d}} T_{14} & \sqrt{\bar{d}} T_{24} & 0
\end{array}\right], \\
& \Theta_{55}^{(p q)}=\left[\begin{array}{ccc}
-\bar{R}_{1 p q} & 0 & 0 \\
* & -e^{-2 \alpha_{2} \tau_{2}} \bar{R}_{2 p q} & e^{-2 \alpha_{2} \tau_{2}} \bar{R}_{2 p q} \\
* & * & -e^{-2 \alpha_{2} \tau_{2}} \bar{R}_{2 p q}
\end{array}\right]
\end{aligned}
$$

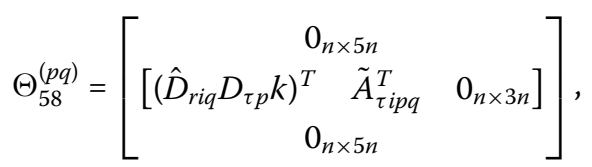

$$
\begin{aligned}
& \Theta_{66}^{(p q)}=\left[\begin{array}{cc}
H e\left(P_{w p q} A_{w}\right)-\alpha_{2} P_{w p q} & -A_{w}^{T} S_{w p q}^{T} \\
* & H e\left(S_{w p q}\right)
\end{array}\right], \quad \Theta_{67}^{(p q)}=\left[\begin{array}{cc}
0_{n \times 3 n} & P_{w p q} B_{w} k^{\prime} \\
0_{n \times 3 n} & -S_{w p q} B_{w} k^{\prime}
\end{array}\right],
\end{aligned}
$$

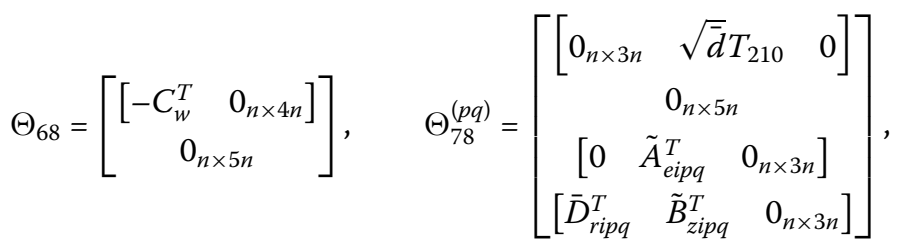

$$
\begin{aligned}
& \Theta_{77}^{(p q)}=\operatorname{diag}\left(H e\left(-2 T_{210} \tilde{E}_{p q}\right)-\bar{d}^{2} \tilde{E}_{p q}^{T} W_{21 p q} \tilde{E}_{p q} \quad H e\left(-2 T_{411} \tilde{E}_{p q}\right) \quad-\Phi_{1 i} \quad-\gamma_{0}^{2} I\right), \\
& \Theta_{88}^{(p q)}=\operatorname{diag}\left(-I-\Delta^{-1} \quad-W_{1} \quad-3 W_{1} \quad-\varepsilon^{-1} \bar{W}_{1}\right) \text {, } \\
& \mathscr{M}_{i p q}=\tilde{E}_{p q}^{T} P_{i p q}+S_{p q} \tilde{R}_{p q}^{T}\left(\tilde{E}_{p q}^{T} \tilde{R}_{p q}=0\right) \text {, } \\
& \Theta_{99}=\operatorname{diag}\left(-\varepsilon_{i 1} \quad \cdots \quad-\varepsilon_{i, i-1} \quad-\varepsilon_{i, i+1} \quad \cdots \quad-\varepsilon_{i, \tilde{k}}\right), \\
& \Delta=\bar{d} W_{1}+d_{1} \bar{W}_{1}+\bar{d}^{2} W_{22 p q}, \\
& \bar{\Theta}_{28}^{(p q)}=\left[\begin{array}{lllll}
\left(\hat{D}_{r i q} D_{p} k\right)^{T} & \tilde{A}_{d i p q}^{T} & \sqrt{\bar{d}} T_{32} & \sqrt{\bar{d}} T_{42} & 0
\end{array}\right], \\
& \bar{\Theta}_{38}^{(p q)}=\left[\begin{array}{lllll}
0 & 0 & \sqrt{\bar{d}} T_{33} & \sqrt{\bar{d}} T_{43} & T_{53}
\end{array}\right], \\
& \bar{\Theta}_{77}^{(p q)}=\operatorname{diag}\left(H e\left(-2 T_{210} \tilde{E}_{p q}\right) \quad H e\left(-2 T_{411} \tilde{E}_{p q}\right)-\bar{d}^{2} \tilde{E}_{p q}^{T} W_{21 p q} \tilde{E}_{p q} \quad-\Phi_{1 i} \quad-\gamma_{0}^{2} I\right) \text {, }
\end{aligned}
$$

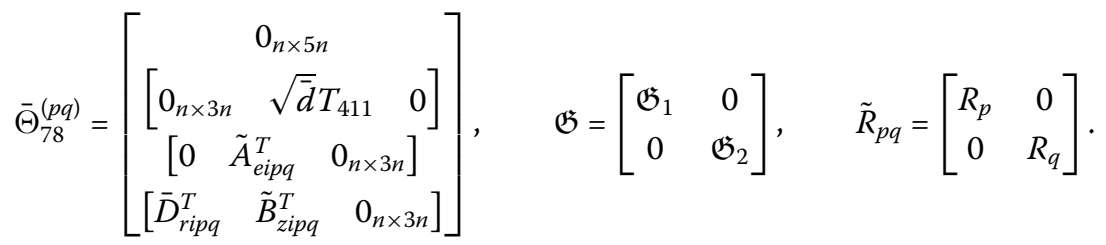

If we replace the switching signal $\tilde{\vartheta}_{t}=q$ and positive scalar $\alpha_{2}$ with switching signal $\vartheta_{t}=p$, and positive scalar $\alpha_{1}$ in the LMIs (19) and (20), we can obtain the LMIs (21) and (22), respectively.

Proof In order to develop our results, we will divide the time interval into two parts, one is $\left[t_{k}, t_{k}+d_{k}\right)(k=0,1, \ldots)$, the other is $\left[t_{k}+d_{k}, t_{k+1}\right)(k=0,1, \ldots)$, which correspond to the asynchronous and synchronous time interval between the switched subsystems and their controller unit, respectively. Next we will discuss our problems in two cases.

Case I: In mismatch period. 
Firstly, we will show that the system (10) is regular and impulse-free. From (19), it is seen that $\Theta_{11}^{(p q)}<0$, so, it is known that there exist two nonsingular matrices $\tilde{M}$ and $\tilde{N}$, such that

$$
\begin{aligned}
& \tilde{M} \tilde{E}_{p q} \tilde{N}=\left[\begin{array}{cc}
I_{2 r} & 0 \\
0 & 0
\end{array}\right], \quad \tilde{M} \tilde{A}_{i p q} \tilde{N}=\left[\begin{array}{cc}
\tilde{A}_{i p q}^{(11)} & \tilde{A}_{i p q}^{(12)} \\
\tilde{A}_{i p q}^{(21)} & \tilde{A}_{i p q}^{(22)}
\end{array}\right], \quad \tilde{M} P_{i p q} \tilde{N}=\left[\begin{array}{cc}
P_{i p q}^{(11)} & P_{i p q}^{(12)} \\
P_{i p q}^{(21)} & P_{i p q}^{(22)}
\end{array}\right], \\
& \tilde{N} S_{p q}=\left[\begin{array}{c}
S_{p q 1} \\
S_{p q 2}
\end{array}\right], \quad \tilde{R}_{p q}=\tilde{M}^{T}\left[\begin{array}{c}
0 \\
\tilde{U}_{p q}^{T}
\end{array}\right],
\end{aligned}
$$

where $\tilde{U}_{p q}$ is any real nonsingular matrix for any $p, q \in \Delta_{1}$. Now, we will pre-multiplying and post-multiplying $\Theta_{11}^{(p q)}<0$ by $\tilde{N}^{T}$ and $\tilde{N}$, we can have $H e\left(S_{p q 2} \tilde{U}_{p q} \tilde{A}_{i p q}^{(22)}\right)<0$, which implies $\tilde{A}_{i p q}^{(22)}$ is nonsingular matrix. Then, for $i \in \mathcal{K}, p, q \in \Delta_{1}$, pair $\left(\tilde{E}_{p q} \tilde{A}_{i p q}\right)$ is regular and impulse-free. Based on Definition 2.2, we can see that augmented system (10) is regular and impulse-free for any time-varying $d(t)$ satisfying Assumption 2.2.

Now, we will show augmented system (10) is SSFTS. Firstly, choose a stochastic Lyapunov function candidate for the system (10) for any $i \in \mathcal{K}, p, q \in \Delta_{1}$ :

$$
\begin{aligned}
& V_{p q}^{(1)}(t)=\tilde{x}^{T}(t) \tilde{E}_{p q}^{T} P_{i p q} \tilde{E}_{p q} \tilde{x}(t), \\
& V_{p q}^{(2)}(t)=\int_{t-d(t)}^{t} e^{\alpha_{2}(t-s)} \tilde{x}^{T}(s) Q_{1 p q} \tilde{x}(s) d s+\int_{t-d_{1}}^{t} e^{\alpha_{2}(t-s)} \tilde{x}^{T}(s) Q_{2 p q} \tilde{x}(s) d s \\
& +\int_{t-d_{2}}^{t} e^{\alpha_{2}(t-s)} \tilde{x}^{T}(s) Q_{3 p q} \tilde{x}(s) d s, \\
& V_{p q}^{(3)}(t)=\tau_{1} \int_{-\tau_{1}}^{0} \int_{t+\theta}^{t} e^{\alpha_{2}(t-s)} \tilde{x}^{T}(s) \bar{R}_{1 p q} \tilde{x}(s) d s d \theta \\
& +\bar{\tau} \int_{-\tau_{2}}^{-\tau_{1}} \int_{t+\theta}^{t} e^{\alpha_{2}(t-s)} \tilde{x}^{T}(s) \bar{R}_{2 p q} \tilde{x}(s) d s d \theta \\
& V_{p q}^{(4)}(t)=\int_{-d_{1}}^{0} \int_{t+\theta}^{t} e^{\alpha_{2}(t-s)} \dot{\tilde{x}}^{T}(s) \tilde{E}_{p q}^{T} \bar{W}_{1 p q} \tilde{E}_{p q} \dot{\tilde{x}}(s) d s d \theta \\
& +\int_{-d_{2}}^{-d_{1}} \int_{t+\theta}^{t} e^{\alpha_{2}(t-s)} \dot{\tilde{x}}^{T}(s) \tilde{E}_{p q}^{T} W_{1 p q} \tilde{E}_{p q} \dot{\tilde{x}}(s) d s d \theta, \\
& V_{p q}^{(5)}(t)=\bar{d} \int_{-d_{2}}^{-d_{1}} \int_{t+\theta}^{t} e^{\alpha_{2}(t-s)}\left[\begin{array}{c}
\tilde{E}_{p q} \tilde{x}(s) \\
\tilde{E}_{p q} \dot{\tilde{x}}(s)
\end{array}\right]^{T}\left[\begin{array}{cc}
W_{21 p q} & 0 \\
0 & W_{22 p q}
\end{array}\right]\left[\begin{array}{c}
\tilde{E}_{p q} \tilde{x}(s) \\
\tilde{E}_{p q} \dot{\tilde{x}}(s)
\end{array}\right] d s d \theta \\
& V_{p q}^{(6)}=\left[\begin{array}{c}
x_{w}(t) \\
\dot{x}_{w}(t)
\end{array}\right]^{T}\left[\begin{array}{cc}
P_{w p q} & 0 \\
0 & S_{w p q}
\end{array}\right]\left[\begin{array}{cc}
I & 0 \\
0 & 0
\end{array}\right]\left[\begin{array}{c}
x_{w}(t) \\
\dot{x}_{w}(t)
\end{array}\right] \text {. }
\end{aligned}
$$

Then the derivative of $V_{p q}(t)$ along the trajectory of the system (10) can be calculated as follows for each $i \in \mathcal{K}, p, q \in \Delta_{1}$ :

$$
\begin{aligned}
\mathscr{L} V_{p q}^{(1)}(t)= & \tilde{x}^{T}(t)\left(H e\left(\mathscr{M}_{i p q} \tilde{A}_{i p q}\right)-\alpha_{2} \tilde{E}_{p q}^{T} P_{i p q} \tilde{E}_{p q}\right) \tilde{x}(t)+2 \tilde{x}^{T}(t) \mathscr{M}_{i p q} \tilde{A}_{\text {dipq }} \tilde{x}(t-d(t)) \\
& +2 \tilde{x}^{T}(t) \mathscr{M}_{i p q} \tilde{A}_{\tau i p q} \int_{t-\tau(t)}^{t} \tilde{x}(s) d s \\
& +2 \tilde{x}^{T}(t) \mathscr{M}_{i p q} \tilde{A}_{e p q} e(t)+2 \tilde{x}^{T}(t) \mathscr{M}_{i p q} \tilde{B}_{z i p q} z(t)+\alpha_{2} V_{p q}^{(1)}(t)+\bar{P}_{i p q}, \\
\mathscr{L} V_{p q}^{(2)}(t) \leq & \alpha_{2} V_{p q}^{(2)}(t)+\tilde{x}^{T}(t)\left(Q_{1 p q}+Q_{2 p q}+Q_{3 p q}\right) \tilde{x}(t)
\end{aligned}
$$




$$
\begin{aligned}
& -(1-d) e^{-\alpha_{2} d_{2}} \tilde{x}^{T}(t-d(t)) Q_{1 p q} \tilde{x}(t-d(t)), \\
\mathscr{L} V_{p q}^{(3)}(t) \leq & \alpha_{2} V_{p q}^{(3)}(t)+\tilde{x}^{T}(t)\left(\tau_{1}^{2} \bar{R}_{1 p q}+\bar{\tau}^{2} \bar{R}_{2 p q}\right) \tilde{x}(t)-\int_{t-\tau_{1}}^{t} \tilde{x}^{T}(s) d s \bar{R}_{1 p q} \int_{t-\tau_{1}}^{t} \tilde{x}(s) d s \\
& -\bar{\tau} e^{-2 \alpha_{2} \tau_{2}} \int_{t-\tau_{2}}^{t-\tau(t)} \tilde{x}^{T}(s) \bar{R}_{2 p q} \tilde{x}(s) d s \\
\leq & \alpha_{2} V_{p q}^{(3)}(t)+\tilde{x}^{T}(t)\left(\tau_{1}^{2} \bar{R}_{1 p q}+\bar{\tau}^{2} \bar{R}_{2 p q}\right) \tilde{x}(t)-\int_{t-\tau_{1}}^{t} \tilde{x}^{T}(s) d s \bar{R}_{1 p q} \int_{t-\tau_{1}}^{t} \tilde{x}(s) d s \\
& -\bar{\tau} e^{-2 \alpha_{2} \tau_{2}}\left[\int_{t-\tau_{2}}^{t} \tilde{x}^{T}(s) d s-\int_{t-\tau(t)}^{t} \tilde{x}^{T}(s) d s\right] \\
& \times \bar{R}_{2 p q}\left[\int_{t-\tau_{2}}^{t} \tilde{x}(s) d s-\int_{t-\tau(t)}^{t} \tilde{x}(s) d s\right], \\
\mathscr{L} V_{p q}^{(4)}(t) \leq & \alpha_{2} V_{p q}^{(4)}(t)+\dot{\tilde{x}}^{T}(t)\left(\bar{d} \tilde{E}_{p q}^{T} W_{1} \tilde{E}_{p q}+d_{1} \tilde{E}_{p q}^{T} \bar{W}_{1} \tilde{E}_{p q}\right) \dot{\tilde{x}}(t) \\
& -\int_{t-d_{2}}^{t-d_{1}} \dot{\tilde{x}}^{T}(s) \tilde{E}_{p q}^{T} W_{1} \tilde{E}_{p q} \dot{\tilde{x}}_{(s) d s} \\
& -\int_{t-d_{1}}^{t} e^{\alpha_{2}(t-s) \dot{\tilde{x}}^{T}(s) \tilde{E}_{p q}^{T} \bar{W}_{1} \tilde{E}_{p q} \dot{\tilde{x}}(s) d s .}
\end{aligned}
$$

Now, from Lemma 2.1 and Lemma 2.2, we have

$$
\begin{aligned}
& -\int_{t-d_{2}}^{t-d_{1}} \dot{\tilde{x}}^{T}(s) \tilde{E}_{p q}^{T} W_{1} \tilde{E}_{p q} \dot{\tilde{x}}(s) d s \\
& \leq \xi^{T}(t)\left[\left(d_{2}-d(t)\right)\left(T_{1} W_{1}^{-1} T_{1}^{T}+\frac{1}{3} T_{2} W_{1}^{-1} T_{2}^{T}\right)\right. \\
& \left.\quad+\left(d(t)-d_{1}\right)\left(T_{3} W_{1}^{-1} T_{3}^{T}+\frac{1}{3} T_{4} W_{1}^{-1} T_{4}^{T}\right)\right] \xi(t) \\
& \quad+\xi^{T}(t) H e\left(T_{1} \tilde{E}_{p q}\left(e_{2}^{T}-e_{4}^{T}\right)\right) \xi(t)+\xi^{T}(t) H e\left(T_{2} \tilde{E}_{p q}\left(e_{2}^{T}+e_{4}^{T}-2 e_{10}^{T}\right)\right) \xi(t) \\
& \quad+\xi^{T}(t) H e\left(T_{3} \tilde{E}_{p q}\left(e_{3}^{T}-e_{2}^{T}\right)\right) \xi(t)+\xi^{T}(t) H e\left(T_{4} \tilde{E}_{p q}\left(e_{3}^{T}+e_{2}^{T}-2 e_{11}^{T}\right)\right) \xi(t) \\
& -\int_{t-d_{1}}^{t} e^{\alpha_{2}(t-s)} \dot{\tilde{x}}^{T}(s) \tilde{E}_{p q}^{T} \bar{W}_{1} \tilde{E}_{p q} \dot{\tilde{x}}^{(s)} d s \\
& \leq \xi^{T}(t) \varepsilon T_{5} \bar{W}_{1}^{-1} T_{5}^{T} \xi(t)+2 \xi \xi^{T}(t) T_{5}\left(\tilde{E}_{p q} \tilde{x}(t)-\tilde{E}_{p q} \tilde{x}\left(t-d_{1}\right)\right),
\end{aligned}
$$

where

$$
\begin{aligned}
& e_{i}^{T}=\left[\begin{array}{lll}
0_{n \times(i-1) n} & I_{n} & 0_{n \times(13-i) n}
\end{array}\right], \quad \varepsilon=\frac{1}{\alpha_{2}}\left(1-e^{-\alpha_{2} d_{1}}\right), \\
& \xi^{T}(t)=\left[\begin{array}{lllll}
\tilde{x}^{T}(t) & \tilde{x}^{T}(t-d(t)) & \tilde{x}^{T}\left(t-d_{1}\right) & \tilde{x}^{T}\left(t-d_{2}\right) & \int_{t-\tau_{1}}^{t} \tilde{x}^{T}(s) d s
\end{array}\right. \\
& \int_{t-\tau(t)}^{t} \tilde{x}^{T}(s) d s \quad \int_{t-\tau_{2}}^{t} \tilde{x}^{T}(s) d s \quad x_{w}^{T}(t) \quad \dot{x}_{w}^{T}(t) \quad \frac{1}{d_{2}-d(t)} \int_{t-d_{2}}^{t-d(t)} \tilde{x}^{T}(s) d s \\
& \left.\frac{1}{d(t)-d_{1}} \int_{t-d(t)}^{t-d_{1}} \tilde{x}^{T}(s) d s \quad e^{T}(t) \quad z^{T}(t)\right], \\
& T_{1}^{T}=\left[\begin{array}{lllllll}
0 & T_{12}^{T} & 0 & T_{14}^{T} & 0 & \cdots & 0
\end{array}\right]_{13 n \times n^{\prime}}, \\
& T_{2}^{T}=\left[\begin{array}{lllllllllll}
0 & T_{22}^{T} & 0 & T_{24}^{T} & 0 & \cdots & 0 & T_{210}^{T} & 0 & 0 & 0
\end{array}\right]_{13 n \times n^{\prime}},
\end{aligned}
$$




$$
\begin{aligned}
T_{3}^{T} & =\left[\begin{array}{llllll}
0 & T_{32}^{T} & T_{33}^{T} & 0 & \cdots & 0
\end{array}\right]_{13 n \times n^{\prime}} \\
T_{4}^{T} & =\left[\begin{array}{lllllllll}
0 & T_{42}^{T} & T_{43}^{T} & 0 & \cdots & 0 & T_{411}^{T} & 0 & 0
\end{array}\right]_{13 n \times n^{\prime}} \\
T_{5}^{T}= & {\left[\begin{array}{llllll}
T_{51}^{T} & 0 & T_{53}^{T} & 0 & \cdots & 0
\end{array}\right]_{13 n \times n^{\prime}} } \\
\mathscr{L} V_{p q}^{(5)}(t) \leq & \alpha_{2} V_{p q}^{(5)}(t)+\bar{d}^{2} \tilde{x}^{T}(t) \tilde{E}_{p q}^{T} W_{21 p q} \tilde{E}_{p q} \tilde{x}(t)+\bar{d}^{2} \dot{\tilde{x}}^{T}(t) \tilde{E}_{p q}^{T} W_{22 p q} \tilde{E}_{p q} \dot{\tilde{x}}(t) \\
& -\bar{d} \int_{t-d_{2}}^{t-d_{1}}\left[\begin{array}{c}
\tilde{E}_{p q} \tilde{x}(s) \\
\tilde{E}_{p q} \dot{\tilde{x}}(s)
\end{array}\right]^{T}\left[\begin{array}{cc}
W_{21 p q} & 0 \\
0 & W_{22 p q}
\end{array}\right]\left[\begin{array}{c}
\tilde{E}_{p q} \tilde{x}(s) \\
\tilde{E}_{p q} \dot{\tilde{x}}(s)
\end{array}\right] d s .
\end{aligned}
$$

In order to improve the feasible region of criteria, for any symmetric matrices $\mathscr{H}_{1}$ and $\mathscr{H}_{2}$, one has

$$
\begin{aligned}
0= & \bar{d}\left(\tilde{x}^{T}(t-d(t)) \tilde{E}_{p q}^{T} \mathscr{H}_{1} \tilde{E}_{p q} \tilde{x}(t-d(t))-\tilde{x}^{T}\left(t-d_{2}\right) \tilde{E}_{p q}^{T} \mathscr{H}_{1} \tilde{E}_{p q} \tilde{x}\left(t-d_{2}\right)\right. \\
& \left.-2 \int_{t-d_{2}}^{t-d(t)} \tilde{x}^{T}(s) \tilde{E}_{p q}^{T} \mathscr{H}_{1} \tilde{E}_{p q} \dot{\tilde{x}}(s) d s\right), \\
0= & \bar{d}\left(\tilde{x}^{T}\left(t-d_{1}\right) \tilde{E}_{p q}^{T} \mathscr{H}_{2} \tilde{E}_{p q} \tilde{x}\left(t-d_{1}\right)-\tilde{x}^{T}(t-d(t)) \tilde{E}_{p q}^{T} \mathscr{H}_{2} \tilde{E}_{p q} \tilde{x}(t-d(t))\right. \\
& \left.-2 \int_{t-d(t)}^{t-d_{1}} \tilde{x}^{T}(s) \tilde{E}_{p q}^{T} \mathscr{H}_{2} \tilde{E}_{p q} \dot{\tilde{x}}(s) d s\right) .
\end{aligned}
$$

Then we have

$$
\begin{aligned}
& -\bar{d} \int_{t-d_{2}}^{t-d(t)}\left[\begin{array}{c}
\tilde{E}_{p q} \tilde{x}(s) \\
\tilde{E}_{p q} \dot{\tilde{x}}(s)
\end{array}\right]^{T}\left[\begin{array}{cc}
W_{21 p q} & 0 \\
0 & W_{22 p q}
\end{array}\right]\left[\begin{array}{c}
\tilde{E}_{p q} \tilde{x}(s) \\
\tilde{E}_{p q} \dot{\tilde{x}}(s)
\end{array}\right] d s \\
& -2 \bar{d} \int_{t-d_{2}}^{t-d(t)} \tilde{x}^{T}(s) \tilde{E}_{p q}^{T} \mathscr{H}_{1} \tilde{E}_{p q} \dot{\tilde{x}}(s) d s \\
& -\bar{d} \int_{t-d(t)}^{t-d_{1}}\left[\begin{array}{c}
\tilde{E}_{p q} \tilde{x}(s) \\
\tilde{E}_{p q} \dot{\tilde{x}}(s)
\end{array}\right]^{T}\left[\begin{array}{cc}
W_{21 p q} & 0 \\
0 & W_{22 p q}
\end{array}\right]\left[\begin{array}{c}
\tilde{E}_{p q} \tilde{x}(s) \\
\tilde{E}_{p q} \dot{\tilde{x}}(s)
\end{array}\right] d s \\
& -2 \bar{d} \int_{t-d(t)}^{t-d_{1}} \tilde{x}^{T}(s) \tilde{E}_{p q}^{T} \mathscr{H}_{2} \tilde{E}_{p q} \dot{\tilde{x}}(s) d s \\
& =-\bar{d} \int_{t-d_{2}}^{t-d(t)}\left[\begin{array}{c}
\tilde{E}_{p q} \tilde{x}(s) \\
\tilde{E}_{p q} \dot{\tilde{x}}(s)
\end{array}\right]^{T}\left[\begin{array}{cc}
W_{21 p q} & \mathscr{H}_{1} \\
\mathscr{H}_{1} & W_{22 p q}
\end{array}\right]\left[\begin{array}{c}
\tilde{E}_{p q} \tilde{x}(s) \\
\tilde{E}_{p q} \dot{\tilde{x}}(s)
\end{array}\right] d s \\
& -\bar{d} \int_{t-d(t)}^{t-d_{1}}\left[\begin{array}{c}
\tilde{E}_{p q} \tilde{x}(s) \\
\tilde{E}_{p q} \dot{\tilde{x}}(s)
\end{array}\right]^{T}\left[\begin{array}{cc}
W_{21 p q} & \mathscr{H}_{2} \\
\mathscr{H}_{2} & W_{22 p q}
\end{array}\right]\left[\begin{array}{c}
\tilde{E}_{p q} \tilde{x}(s) \\
\tilde{E}_{p q} \dot{\tilde{x}}(s)
\end{array}\right] d s \\
& \leq-\frac{\bar{d}}{d_{2}-d(t)} \xi^{T}(t)\left[\left(d_{2}-d(t)\right) e_{10} \tilde{E}_{p q}^{T} \quad e_{2} \tilde{E}_{p q}^{T}-e_{4} \tilde{E}_{p q}^{T}\right] \\
& \times\left[\begin{array}{cc}
W_{21 p q} & \mathscr{H}_{1} \\
\mathscr{H}_{1} & W_{22 p q}
\end{array}\right]\left[\begin{array}{c}
\left(d_{2}-d(t)\right) \tilde{E}_{p q} e_{10}^{T} \\
\tilde{E}_{p q} e_{2}^{T}-\tilde{E}_{p q} e_{4}^{T}
\end{array}\right] \xi(t) \\
& -\frac{\bar{d}}{d(t)-d_{1}} \xi^{T}(t)\left[\left(d(t)-d_{1}\right) e_{11} \tilde{E}_{p q}^{T} \quad e_{3} \tilde{E}_{p q}^{T}-e_{2} \tilde{E}_{p q}^{T}\right]
\end{aligned}
$$




$$
\begin{aligned}
& \times\left[\begin{array}{cc}
W_{21 p q} & \mathscr{H}_{2} \\
\mathscr{H}_{2} & W_{22 p q}
\end{array}\right]\left[\begin{array}{c}
\left(d(t)-d_{1}\right) \tilde{E}_{p q} e_{11}^{T} \\
\tilde{E}_{p q} e_{3}^{T}-\tilde{E}_{p q} e_{2}^{T}
\end{array}\right] \xi(t) \\
= & \xi^{T}(t)\left(-\bar{d}\left(\mathfrak{F}_{1}+\frac{1}{d_{2}-d(t)} \mathfrak{F}_{2}\right)\right)\left[\begin{array}{cc}
W_{21 p q} & \mathscr{H}_{1} \\
\mathscr{H}_{1} & W_{22 p q}
\end{array}\right]\left(\left(d_{2}-d(t)\right) \mathfrak{F}_{1}^{T}+\mathfrak{F}_{2}^{T}\right) \xi(t) \\
& +\xi^{T}(t)\left(-\bar{d}\left(\mathfrak{F}_{3}+\frac{1}{d(t)-d_{1}} \mathfrak{F}_{4}\right)\right)\left[\begin{array}{cc}
W_{21 p q} & \mathscr{H}_{2} \\
\mathscr{H}_{2} & W_{22 p q}
\end{array}\right]\left(\left(d(t)-d_{1}\right) \mathfrak{F}_{3}^{T}+\mathfrak{F}_{4}^{T}\right) \xi(t),
\end{aligned}
$$

where

$$
\begin{array}{ll}
\mathfrak{F}_{1}=\left[\begin{array}{ll}
e_{10} \tilde{E}_{p q}^{T} & 0
\end{array}\right], & \mathfrak{F}_{2}=\left[\begin{array}{ll}
0 & e_{2} \tilde{E}_{p q}^{T}-e_{4} \tilde{E}^{T}
\end{array}\right], \\
\mathfrak{F}_{3}=\left[\begin{array}{ll}
e_{11} \tilde{E}_{p q}^{T} & 0
\end{array}\right], & \mathfrak{F}_{4}=\left[\begin{array}{ll}
0 & e_{3} \tilde{E}_{p q}^{T}-e_{2} \tilde{E}_{p q}^{T}
\end{array}\right] .
\end{array}
$$

Based on the reciprocally convex approach, for any matrix $\mathfrak{G}$, we can obtain the following inequality:

$$
\begin{aligned}
& -\frac{\bar{d}}{d_{2}-d(t)} \mathfrak{F}_{2}\left[\begin{array}{cc}
W_{21 p q} & \mathscr{H}_{1} \\
\mathscr{H}_{1} & W_{22 p q}
\end{array}\right] \mathfrak{F}_{2}^{T}-\frac{\bar{d}}{d(t)-d_{1}} \mathfrak{F}_{4}\left[\begin{array}{cc}
W_{21 p q} & \mathscr{H}_{2} \\
\mathscr{H}_{2} & W_{22 p q}
\end{array}\right] \mathfrak{F}_{4}^{T} \\
& \leq-\left[\begin{array}{ll}
\mathfrak{F}_{2} & \mathfrak{F}_{4}
\end{array}\right]\left[\begin{array}{cc}
{\left[\begin{array}{cc}
W_{21 p q} & \mathscr{H}_{1} \\
\mathscr{H}_{1} & W_{22 p q}
\end{array}\right]} & \mathfrak{G} \\
\mathfrak{G}^{T} & {\left[\begin{array}{cc}
W_{21 p q} & \mathscr{H}_{2} \\
\mathscr{H}_{2} & W_{22 p q}
\end{array}\right]}
\end{array}\right]\left[\begin{array}{c}
\mathfrak{F}_{2}^{T} \\
\mathfrak{F}_{4}^{T}
\end{array}\right], \\
& \mathscr{L} V_{p q}^{(6)}(t)=2\left[\begin{array}{c}
x_{w}(t) \\
\dot{x}_{w}(t)
\end{array}\right]^{T}\left[\begin{array}{cc}
P_{w p q} & 0 \\
0 & S_{w p q}
\end{array}\right]\left[\begin{array}{c}
\dot{x}_{w}(t) \\
\dot{x}_{w}(t)-A_{w} x_{w}(t)-B_{w} k^{\prime} z(t)
\end{array}\right] \\
& =x_{w}^{T}(t)\left(H e\left(P_{w p q} A_{w}\right)-\alpha_{2} P_{w p q}\right) x_{w}(t)-2 x_{w}^{T}(t) A_{w}^{T} S_{w p q}^{T} \dot{x}_{w}(t) \\
& +2 x_{w}^{T}(t) P_{w p q} B_{w} k^{\prime} z(t)+\dot{x}_{w}^{T}(t)\left(H e\left(S_{w p q}\right)\right) \dot{x}_{w}(t) \\
& -2 \dot{x}_{w}^{T}(t) S_{w p q} B_{w} k^{\prime} z(t)+\alpha_{2} V_{p q}^{(6)}(t) \text {. }
\end{aligned}
$$

Now, we will analyze the uncertain transition rate $\pi_{i j}^{(p q)}$ with the assumption (6) as follows:

$$
\begin{aligned}
\bar{P}_{i p q}= & \sum_{j=1}^{\tilde{k}} \pi_{i j}^{(p q)} \tilde{E}_{p q}^{T} P_{j p q} \tilde{E}_{p q}=\sum_{\substack{j=1 \\
j \neq i}}^{\tilde{k}} \pi_{i j}^{(p q)} \tilde{E}_{p q}^{T} P_{j p q} \tilde{E}_{p q}+\pi_{i i}^{(p q)} \tilde{E}_{p q}^{T} P_{i p q} \tilde{E}_{p q} \\
= & \sum_{\substack{j=1 \\
j \neq i}}^{\tilde{k}} \pi_{i j}^{(p q)} \tilde{E}_{p q}^{T}\left(P_{j p q}-P_{i p q}\right) \tilde{E}_{p q}=\sum_{\substack{j=1 \\
j \neq i}}^{\tilde{k}}\left(\bar{\pi}_{i j}^{(p q)}+\Delta \bar{\pi}_{i j}^{(p q)}\right) \tilde{E}_{p q}^{T}\left(P_{j p q}-P_{i p q}\right) \tilde{E}_{p q} \\
\leq & \sum_{\substack{j=1 \\
j \neq i}}^{\tilde{k}} \bar{\pi}_{i j}^{(p q)} \tilde{E}_{p q}^{T}\left(P_{j p q}-P_{i p q}\right) \tilde{E}_{p q}+\sum_{\substack{j=1 \\
j \neq i}}^{\tilde{k}} \frac{1}{4}\left(\delta_{i j}^{(p q)}\right)^{2} \varepsilon_{i j} \\
& +\tilde{E}_{p q}^{T}\left(P_{j p q}-P_{i p q}\right) \tilde{E}_{p q} \varepsilon_{i j}^{-1} \tilde{E}_{p q}^{T}\left(P_{j p q}-P_{i p q}\right) \tilde{E}_{p q} .
\end{aligned}
$$


Next, in order to deduce our results, we suppose that

$$
\Upsilon(t)=\gamma_{0}^{2} z^{T}(t) z(t)-\hat{r}^{T}(t) \hat{r}(t)
$$

In view of event condition (9), $e^{T}(t) \Phi_{1 i} e(t)-\rho_{i} x_{f}^{T}(t) \Phi_{2 i} x_{f}(t) \leq 0$ holds for all $t \geq 0$ and combining the above discussion by the Schur complement lemma, it can be deduced that

$$
L V_{p q}(t)-\alpha_{2} V_{p q}(t)-\Upsilon(t)<0
$$

if inequalities (19) and (20) are satisfied.

Case II: In match period.

For $t \in\left[t_{k}+d_{k}, t_{k+1}\right)(k=0,1, \ldots)$, which is the synchronous time interval between the switched subsystems and their controller unit. Next, similar to Case I, we will further analyze our results under the match period. Let choose the following multiple Lyapunov-like functional for the augmented system (10) as

$$
\begin{aligned}
& V_{p}^{(1)}(t)=\tilde{x}^{T}(t) \tilde{E}_{p}^{T} P_{i p} \tilde{E}_{p} \tilde{x}(t) \\
& V_{p}^{(2)}(t)=\int_{t-d(t)}^{t} e^{\alpha_{1}(t-s)} \tilde{x}^{T}(s) Q_{1 p} \tilde{x}(s) d s+\int_{t-d_{1}}^{t} e^{\alpha_{1}(t-s)} \tilde{x}^{T}(s) Q_{2 p} \tilde{x}(s) d s \\
& +\int_{t-d_{2}}^{t} e^{\alpha_{1}(t-s)} \tilde{x}^{T}(s) Q_{3 p} \tilde{x}(s) d s, \\
& V_{p}^{(3)}(t)=\tau_{1} \int_{-\tau_{1}}^{0} \int_{t+\theta}^{t} e^{\alpha_{1}(t-s)} \tilde{x}^{T}(s) \bar{R}_{1 p} \tilde{x}(s) d s d \theta \\
& +\bar{\tau} \int_{-\tau_{2}}^{-\tau_{1}} \int_{t+\theta}^{t} e^{\alpha_{1}(t-s)} \tilde{x}^{T}(s) \bar{R}_{2 p} \tilde{x}(s) d s d \theta, \\
& V_{p}^{(4)}(t)=\int_{-d_{1}}^{0} \int_{t+\theta}^{t} e^{\alpha_{1}(t-s)} \dot{\tilde{x}}^{T}(s) \tilde{E}_{p}^{T} \bar{W}_{1 p} \tilde{E}_{p} \dot{\tilde{x}}(s) d s d \theta \\
& +\int_{-d_{2}}^{-d_{1}} \int_{t+\theta}^{t} e^{\alpha_{1}(t-s)} \dot{\tilde{x}}^{T}(s) \tilde{E}_{p}^{T} W_{1 p} \tilde{E}_{p} \dot{\tilde{x}}(s) d s d \theta \\
& V_{p}^{(5)}(t)=\bar{d} \int_{-d_{2}}^{-d_{1}} \int_{t+\theta}^{t} e^{\alpha_{1}(t-s)}\left[\begin{array}{c}
\tilde{E}_{p} \tilde{x}(s) \\
\tilde{E}_{p} \dot{\tilde{x}}(s)
\end{array}\right]^{T}\left[\begin{array}{cc}
W_{21 p} & 0 \\
0 & W_{22 p}
\end{array}\right]\left[\begin{array}{c}
\tilde{E}_{p} \tilde{x}(s) \\
\tilde{E}_{p} \dot{\tilde{x}}(s)
\end{array}\right] d s d \theta, \\
& V_{p q}^{(6)}=\left[\begin{array}{c}
x_{w}(t) \\
\dot{x}_{w}(t)
\end{array}\right]^{T}\left[\begin{array}{cc}
P_{w p} & 0 \\
0 & S_{w p}
\end{array}\right]\left[\begin{array}{cc}
I & 0 \\
0 & 0
\end{array}\right]\left[\begin{array}{c}
x_{w}(t) \\
\dot{x}_{w}(t)
\end{array}\right] \text {. }
\end{aligned}
$$

Similar to the proof of Case I, if (21) and (22) hold, then

$$
L V_{p}(t)-\alpha_{1} V_{p}(t)-\Upsilon(t)<0 .
$$

Then from (37) and (44), we can have

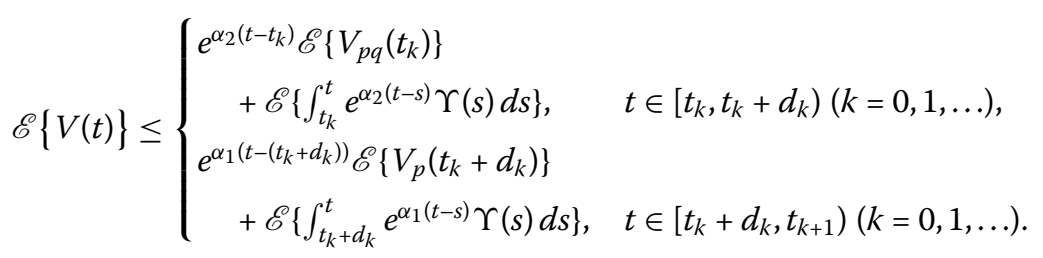


Step 1. Firstly, we will show that the augmented system (10) is SSFTS under the conditions of Theorem 3.1 if $z(t)=0$.

Based on the truth of (37) and (44), we know that if $z(t)=0$, (45) is equivalent to

$$
\mathscr{E}\{V(t)\} \leq \begin{cases}e^{\alpha_{2}\left(t-t_{k}\right)} \mathscr{E}\left\{V_{p q}\left(t_{k}\right)\right\}, & t \in\left[t_{k}, t_{k}+d_{k}\right)(k=0,1, \ldots), \\ e^{\alpha_{1}\left(t-\left(t_{k}+d_{k}\right)\right)} \mathscr{E}\left\{V_{p}\left(t_{k}+d_{k}\right)\right\}, & t \in\left[t_{k}+d_{k}, t_{k+1}\right)(k=0,1, \ldots) .\end{cases}
$$

Without loss of generality, one assumes that $\alpha_{1} \geq \alpha_{2}$ and the other condition of $\alpha_{1}<\alpha_{2}$ will be analyzed in the following.

From the definitions of $V_{p q}(t)$ and $V_{p}(t)$, it shows that if $\alpha_{1} \geq \alpha_{2}$,

$$
\mathscr{E}\left\{V_{p q}(t)\right\} \leq \mu_{1} E\left\{V_{p}(t)\right\} \quad E\left\{V_{p}(t)\right\} \leq \mu_{2} e^{d_{\tau}\left(\alpha_{1}-\alpha_{2}\right)} \mathscr{E}\left\{V_{p q}(t)\right\}
$$

where

$$
\begin{aligned}
& \mu_{1}=\max \left\{\frac{\max _{p, q \in \Delta_{1}, i \in \tilde{k}}\left(\lambda_{\max }\left(\tilde{E}_{p q}^{T} P_{i p q} \tilde{E}_{p q}\right)\right)}{\min _{p \in \Delta_{1}, i \in \tilde{k}}\left(\lambda_{\min }\left(\tilde{E}_{p}^{T} P_{i p} \tilde{E}_{p}\right)\right)}, \frac{\max _{p, q \in \Delta_{1}}\left(\lambda_{\max }\left(P_{w p q}\right)\right)}{\min _{p \in \Delta_{1}}\left(\lambda_{\min }\left(P_{w p}\right)\right)},\right. \\
& \frac{\max _{p, q \in \Delta_{1}}\left(\lambda_{\max }\left(Q_{1 p q}\right)\right)}{\min _{p \in \Delta_{1}}\left(\lambda_{\min }\left(Q_{1 p}\right)\right)}, \frac{\max _{p, q \in \Delta_{1}}\left(\lambda_{\max }\left(Q_{2 p q}\right)\right)}{\min _{p \in \Delta_{1}}\left(\lambda_{\min }\left(Q_{2 p}\right)\right)}, \frac{\max _{p, q \in \Delta_{1}}\left(\lambda_{\max }\left(Q_{3 p q}\right)\right)}{\min _{p \in \Delta_{1}}\left(\lambda_{\min }\left(Q_{3 p}\right)\right)}, \\
& \frac{\max _{p, q \in \Delta_{1}}\left(\lambda_{\max }\left(\bar{R}_{1 p q}\right)\right)}{\min _{p \in \Delta_{1}}\left(\lambda_{\min }\left(\bar{R}_{1 p}\right)\right)}, \frac{\max _{p, q \in \Delta_{1}}\left(\lambda_{\max }\left(\bar{R}_{2 p q}\right)\right)}{\min _{p \in \Delta_{1}}\left(\lambda_{\min }\left(\bar{R}_{2 p}\right)\right)}, \frac{\max _{p, q \in \Delta_{1}}\left(\lambda_{\max }\left(\tilde{E}_{p q}^{T} W_{1 p q} \tilde{E}_{p q}\right)\right)}{\min _{p \in \Delta_{1}}\left(\lambda_{\min }\left(\tilde{E}_{p}^{T} W_{1 p} \tilde{E}_{p}\right)\right)}, \\
& \frac{\max _{p, q \in \Delta_{1}}\left(\lambda_{\max }\left(\tilde{E}_{p q}^{T} \bar{W}_{1 p q} \tilde{E}_{p q}\right)\right)}{\min _{p \in \Delta_{1}}\left(\lambda_{\min }\left(\tilde{E}_{p}^{T} \bar{W}_{1 p} \tilde{E}_{p}\right)\right)}, \frac{\max _{p, q \in \Delta_{1}}\left(\lambda_{\max }\left(\tilde{E}_{p q}^{T} W_{21 p q} \tilde{E}_{p q}\right)\right)}{\min _{p \in \Delta_{1}}\left(\lambda_{\min }\left(\tilde{E}_{p}^{T} W_{21 p} \tilde{E}_{p}\right)\right)}, \\
& \left.\frac{\max _{p, q \in \Delta_{1}}\left(\lambda_{\max }\left(\tilde{E}_{p q}^{T} W_{22 p q} \tilde{E}_{p q}\right)\right)}{\min _{p \in \Delta_{1}}\left(\lambda_{\min }\left(\tilde{E}_{p}^{T} W_{22 p} \tilde{E}_{p}\right)\right)}\right\} \\
& \mu_{2}=\max \left\{\frac{\max _{p \in \Delta_{1}, i \in \tilde{k}}\left(\lambda_{\max }\left(\tilde{E}_{p}^{T} P_{i p} \tilde{E}_{p}\right)\right)}{\min _{p, q \in \Delta_{1}, i \in \tilde{k}}\left(\lambda_{\min }\left(\tilde{E}_{p q}^{T} P_{i p q} \tilde{E}_{p q}\right)\right)}, \frac{\max _{p \in \Delta_{1}}\left(\lambda_{\max }\left(P_{w p}\right)\right)}{\min _{p, q \in \Delta_{1}}\left(\lambda_{\min }\left(P_{w p q}\right)\right)},\right. \\
& \frac{\max _{p \in \Delta_{1}}\left(\lambda_{\max }\left(Q_{1 p}\right)\right)}{\min _{p, q \in \Delta_{1}}\left(\lambda_{\min }\left(Q_{1 p q}\right)\right)}, \frac{\max _{p \in \Delta_{1}}\left(\lambda_{\max }\left(Q_{2 p}\right)\right)}{\min _{p, q \in \Delta_{1}}\left(\lambda_{\min }\left(Q_{2 p q}\right)\right)}, \frac{\max _{p \in \Delta_{1}}\left(\lambda_{\max }\left(Q_{3 p}\right)\right)}{\min _{p, q \in \Delta_{1}}\left(\lambda_{\min }\left(Q_{3 p q}\right)\right)}, \\
& \frac{\max _{p \in \Delta_{1}}\left(\lambda_{\max }\left(\bar{R}_{1 p}\right)\right)}{\min _{p, q \in \Delta_{1}}\left(\lambda_{\min }\left(\bar{R}_{1 p q}\right)\right)}, \frac{\max _{p \in \Delta_{1}}\left(\lambda_{\max }\left(\bar{R}_{2 p}\right)\right)}{\min _{p, q \in \Delta_{1}}\left(\lambda_{\min }\left(\bar{R}_{2 p q}\right)\right)}, \frac{\max _{p \in \Delta_{1}}\left(\lambda_{\max }\left(\tilde{E}_{p}^{T} W_{1 p} \tilde{E}_{p}\right)\right)}{\min _{p, q \in \Delta_{1}}\left(\lambda_{\min }\left(\tilde{E}_{p q}^{T} W_{1 p q} \tilde{E}_{p q}\right)\right)}, \\
& \frac{\max _{p \in \Delta_{1}}\left(\lambda_{\max }\left(\tilde{E}_{p}^{T} \bar{W}_{1 p} \tilde{E}_{p}\right)\right)}{\min _{p, q \in \Delta_{1}}\left(\lambda_{\min }\left(\tilde{E}_{p q}^{T} \bar{W}_{1 p q} \tilde{E}_{p q}\right)\right)}, \frac{\max _{p \in \Delta_{1}}\left(\lambda_{\max }\left(\tilde{E}_{p}^{T} W_{21 p} \tilde{E}_{p}\right)\right)}{\min _{p, q \in \Delta_{1}}\left(\lambda_{\min }\left(\tilde{E}_{p q}^{T} W_{21 p q} \tilde{E}_{p q}\right)\right)}, \\
& \left.\frac{\max _{p \in \Delta_{1}}\left(\lambda_{\max }\left(\tilde{E}_{p}^{T} W_{22 p} \tilde{E}_{p}\right)\right)}{\min _{p, q \in \Delta_{1}}\left(\lambda_{\min }\left(\tilde{E}_{p q}^{T} W_{22 p q} \tilde{E}_{p q}\right)\right)}\right\} \text {. }
\end{aligned}
$$

Then, for any $t \in\left[t_{k}, t_{k}+d_{k}\right)$ and $\alpha_{1} \geq \alpha_{2}$, we can obtain

$$
\begin{aligned}
\mathscr{E}\{V(t)\} & \leq e^{\alpha_{2}\left(t-t_{k}\right)} \mathscr{E}\left\{V_{p q}\left(t_{k}\right)\right\} \leq \mu_{1} e^{\alpha_{2}\left(t-t_{k}\right)} \mathscr{E}\left\{V_{p}\left(t_{k}^{-}\right)\right\} \\
& \leq \mu_{1} e^{\alpha_{2}\left(t-t_{k}\right)} e^{\alpha_{1}\left(t_{k}-\left(t_{k-1}+d_{k-1}\right)\right)} \mathscr{E}\left\{V_{p}\left(t_{k-1}+d_{k-1}\right)\right\} \\
& \leq \mu_{1} e^{\alpha_{2}\left(t-t_{k}\right)} e^{\alpha_{1}\left(t_{k}-\left(t_{k-1}+d_{k-1}\right)\right)} \mu_{2} e^{d_{\tau}\left(\alpha_{1}-\alpha_{2}\right)} \mathscr{E}\left\{V_{p q}\left(t_{k-1}+d_{k-1}\right)^{-}\right\}
\end{aligned}
$$




$$
\begin{aligned}
& \leq \mu_{1} e^{\alpha_{2}\left(t-t_{k}\right)} e^{\alpha_{1}\left(t_{k}-\left(t_{k-1}+d_{k-1}\right)\right)} \mu_{2} e^{d_{\tau}\left(\alpha_{1}-\alpha_{2}\right)} e^{\alpha_{2}\left(t_{k-1}+d_{k-1}-t_{k-1}\right)} \mathscr{E}\left\{V_{p q}\left(t_{k-1}\right)\right\} \\
& \leq \cdots \\
& \leq \mu_{1}^{N_{\vartheta}\left(t_{0}, t\right)} \mu_{2}^{N_{\tilde{\vartheta}}\left(t_{0}, t\right)} e^{N_{\tilde{\vartheta}\left(t_{0}, t\right)} d_{\tau}\left(\alpha_{1}-\alpha_{2}\right)} e^{\alpha_{2}\left(t-t_{k}+d_{k-1}+d_{k-2}+\cdots+d_{0}\right)} \\
& \times e^{\alpha_{1}\left(t_{k}-d_{k-1}-d_{k-2}-\cdots-d_{0}-t_{0}\right)} \mathscr{E}\left\{V\left(t_{0}\right)\right\} .
\end{aligned}
$$

It is easy to obtain the following fact through simple calculations:

$$
\begin{aligned}
& t-t_{k}+d_{k-1}+d_{k-2}+\cdots+d_{0}<d_{k}+d_{k-1}+d_{k-2}+\cdots+d_{0}=T_{\mathrm{mism}}\left(t_{0}, t\right), \\
& t_{k}-d_{k-1}-d_{k-2}-\cdots-d_{0}-t_{0} \\
& \quad=\left(t_{k}-t_{k-1}-d_{k-1}\right)+\left(t_{k-1}-t_{k-2}-d_{k-2}\right)+\cdots+\left(t_{1}-t_{0}-d_{0}\right)=T_{\mathrm{m}}\left(t_{0}, t\right),
\end{aligned}
$$

where $T_{\text {mism }}\left(t_{0}, t\right)$ and $T_{\mathrm{m}}\left(t_{0}, t\right)$ denote the total mismatched time and matched time interval lengths on $\left[t_{0}, t\right] . N_{\vartheta\left(t_{0}, t\right)}$ and $N_{\tilde{\vartheta}\left(t_{0}, t\right)}$ denote the number of synchronous and asynchronous switching on the interval $\left[t_{0}, t\right)$, respectively. Furthermore, based on the assumption of $d_{\text {max }}$, we know that relational expression $N_{\tilde{\vartheta}\left(t_{0}, t\right)}<N_{\vartheta\left(t_{0}, t\right)}$ is true.

In conclusion, it gives that

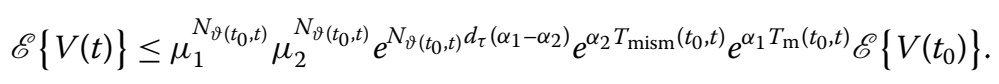

Next, if $\alpha_{1}<\alpha_{2}$, we can also obtain following inequalities very easily by the similar analysis in (47):

$$
\mathscr{E}\left\{V_{p}(t)\right\} \leq \mu_{2} \mathscr{E}\left\{V_{p q}(t)\right\}, \quad \mathscr{E}\left\{V_{p q}(t)\right\} \leq \mu_{1} e^{d_{\tau}\left(\alpha_{2}-\alpha_{1}\right)} \mathscr{E}\left\{V_{p}(t)\right\}
$$

According to the same techniques as in the proof of (52), the following inequality is true

$$
\mathscr{E}\{V(t)\} \leq \mu_{1}^{N_{\vartheta\left(t_{0}, t\right)}} \mu_{2}^{N_{\vartheta\left(t_{0}, t\right)}} e^{N_{\vartheta\left(t_{0}, t\right)} d_{\tau}\left(\alpha_{2}-\alpha_{1}\right)} e^{\alpha_{2} T_{\mathrm{mism}}\left(t_{0}, t\right)} e^{\alpha_{1} T_{\mathrm{m}}\left(t_{0}, t\right)} \mathscr{E}\left\{V\left(t_{0}\right)\right\} .
$$

By combining the two cases with $\alpha_{1} \geq \alpha_{2}$ and $\alpha_{1}<\alpha_{2}$, eventually, we have the estimation of $\mathscr{E}\{V(t)\}$ as follows:

$$
\mathscr{E}\{V(t)\} \leq \mu_{1}^{N_{\vartheta\left(t_{0}, t\right)}} \mu_{2}^{N_{\vartheta\left(t_{0}, t\right)}} e^{N_{\vartheta\left(t_{0}, t\right)} d_{\tau}\left|\alpha_{1}-\alpha_{2}\right|} e^{\alpha_{2} T_{\mathrm{mism}}\left(t_{0}, t\right)} e^{\alpha_{1} T_{\mathrm{m}}\left(t_{0}, t\right)} \mathscr{E}\left\{V\left(t_{0}\right)\right\} .
$$

According to the definition of $V(t)$, we know that

$$
\mathscr{E}\{V(t)\} \geq \eta_{1} \mathscr{E}\left\{\tilde{x}^{T}(t) \mathscr{G} \tilde{x}(t)\right\}
$$

where

$$
\eta_{1}=\min \left\{\frac{\min _{p, q \in \Delta_{1}, i \in \tilde{k}}\left(\lambda_{\min }\left(\tilde{E}_{p q}^{T} P_{i p q} \tilde{E}_{p q}\right)\right)}{\lambda_{\max }(\mathscr{G})}, \frac{\min _{p \in \Delta_{1}, i \in \tilde{k}}\left(\lambda_{\min }\left(\tilde{E}_{p}^{T} P_{i p} \tilde{E}_{p}\right)\right)}{\lambda_{\max }(\mathscr{G})}\right\}
$$

and

$$
\mathscr{E}\left\{V\left(t_{0}\right)\right\} \leq \eta_{2} c_{2}
$$


where $\bar{\alpha}=\max \left\{\alpha_{1}, \alpha_{2}\right\}$,

$$
\begin{aligned}
& \eta_{2}=\left\{\frac{\max _{p, q \in \Delta_{1}, i \in \tilde{k}}\left(\lambda_{\max }\left(\tilde{E}_{p q}^{T} P_{i p q} \tilde{E}_{p q}\right)\right)}{\lambda_{\min }(\mathscr{G})}\right. \\
& +\frac{e^{\bar{\alpha} d_{2}}\left(\max _{p, q \in \Delta_{1}}\left(\lambda_{\max }\left(Q_{1 p q}\right)\right)+\max _{p, q \in \Delta_{1}}\left(\lambda_{\max }\left(Q_{3 p q}\right)\right)\right)}{\lambda_{\min }(\mathscr{G})} \\
& +\frac{e^{\bar{\alpha} d_{1}}\left(\max _{p, q \in \Delta_{1}}\left(\lambda_{\max }\left(Q_{2 p q}\right)\right)\right)}{\lambda_{\min }(\mathscr{G})}+\frac{e^{\bar{\alpha} \tau_{1}} \tau_{1}^{2} \max _{p, q \in \Delta_{1}}\left(\lambda_{\max }\left(\bar{R}_{1 p q}\right)\right)}{\lambda_{\min }(\mathscr{G})} \\
& +\frac{e^{\bar{\alpha} \tau_{2}} \bar{\tau}^{2} \max _{p, q \in \Delta_{1}}\left(\lambda_{\max }\left(\bar{R}_{2 p q}\right)\right)}{\lambda_{\min }(\mathscr{G})}+\frac{e^{\bar{\alpha} d_{2}} \bar{d}^{2} \max _{p, q \in \Delta_{1}}\left(\lambda_{\max }\left(\tilde{E}_{p q}^{T} W_{21 p q} \tilde{E}_{p q}\right)\right)}{\lambda_{\min }(\mathscr{G})} \\
& +\frac{e^{\bar{\alpha} d_{2}} \bar{d} \max _{p, q \in \Delta_{1}}\left(\lambda_{\max }\left(\tilde{E}_{p q}^{T} W_{1 p q} \tilde{E}_{p q}\right)\right)}{\lambda_{\min }(\mathscr{G})}+\frac{\max _{p, q \in \Delta_{1}}\left(\lambda_{\max }\left(P_{w p q}\right)\right)}{\lambda_{\min }(\mathscr{G})} \\
& +\frac{e^{\bar{\alpha} d_{1}} d_{1} \max _{p, q \in \Delta_{1}}\left(\lambda_{\max }\left(\tilde{E}_{p q}^{T} \bar{W}_{1 p q} \tilde{E}_{p q}\right)\right)}{\lambda_{\min }(\mathscr{G})} \\
& \left.+\frac{e^{\bar{\alpha} d_{2}} \bar{d}^{2} \max _{p, q \in \Delta_{1}}\left(\lambda_{\max }\left(\tilde{E}_{p q}^{T} W_{22 p q} \tilde{E}_{p q}\right)\right)}{\lambda_{\min }(\mathscr{G})}\right\} \text {. }
\end{aligned}
$$

Then for any $t \in\left[t_{0}, T\right]$, we can conclude that

$$
\mathscr{E}\left\{\tilde{x}^{T}(t) \mathscr{G} \tilde{x}(t)\right\} \leq \frac{\left(\mu_{1} \mu_{2}\right)^{\frac{T}{\tau_{a}}} e^{\frac{T}{\tau_{a}}\left|\alpha_{1}-\alpha_{2}\right| d_{\tau}} e^{\alpha_{1} T_{\mathrm{m}}\left(t_{0}, T\right)+\alpha_{2} T_{\mathrm{mism}}\left(t_{0}, T\right)} \eta_{2} c_{1}}{\eta_{1}}
$$

Therefore, from (24), (25), we can have $\mathscr{E}\left\{\tilde{x}^{T}(t) \mathscr{G} \tilde{x}(t)\right\}<c_{2}$, then, according to Definition 2.3, system (10) is SSFTS under the asynchronous switching.

Step 2 . In this section, we will show that $H_{\infty}$ property (12) should be guaranteed if the conditions of Theorem 3.1 are satisfied.

Firstly, according to the (45) and the similar proof of Step 1, we can conclude that

$$
\begin{aligned}
\mathscr{E}\{V(t)\} \leq & \left(\mu_{1} \mu_{2} e^{\left|\alpha_{1}-\alpha_{2}\right| d_{\tau}}\right)^{N_{\vartheta}\left(t_{0}, t\right)} e^{\alpha_{1} T_{\mathrm{m}}\left(t_{0}, t\right)+\alpha_{2} T_{\mathrm{mism}}\left(t_{0}, t\right)} \mathscr{E}\left\{V\left(t_{0}\right)\right\} \\
& \times \mathscr{E}\left\{\int_{t_{0}}^{t}\left(\mu_{1} \mu_{2} e^{\left|\alpha_{1}-\alpha_{2}\right| d_{\tau}}\right)^{N_{\vartheta}(s, t)} e^{\alpha_{1} T_{\mathrm{m}}(s, t)+\alpha_{2} T_{\mathrm{mism}}(s, t)} \Upsilon(s) d s\right\} .
\end{aligned}
$$

From $\mathscr{E}\{V(t)\} \geq 0,(60)$ implies that under zero initial conditions and for $t_{0}=0$

$$
\begin{aligned}
& \mathscr{E}\left\{\int_{0}^{t}\left(e^{\ln \left(\mu_{1} \mu_{2}+e^{\left|\alpha_{1}-\alpha_{2}\right| d_{\tau}}\right)}\right)^{N_{\vartheta}(s, t)} e^{\alpha_{1} T_{\mathrm{m}}(s, t)+\alpha_{2} T_{\mathrm{mism}}(s, t)} \hat{r}^{T}(s) \hat{r}(s) d s\right\} \\
& \leq \gamma_{0}^{2} \mathscr{E}\left\{\int_{0}^{t}\left(e^{\ln \left(\mu_{1} \mu_{2}+e^{\left|\alpha_{1}-\alpha_{2}\right| d_{\tau}}\right)}\right)^{N_{\vartheta}(s, t)} e^{\alpha_{1} T_{\mathrm{m}}(s, t)+\alpha_{2} T_{\mathrm{mism}}(s, t)} z^{T}(s) z(s) d s\right\} \\
& \leq \gamma_{0}^{2} \mathscr{E}\left\{\int_{0}^{t}\left(e^{\ln \left(\mu_{1} \mu_{2}+e^{\left|\alpha_{1}-\alpha_{2}\right| d_{\tau}}\right)}\right)^{N_{\vartheta}(s, t)} e^{\alpha_{1}(t-s)+\alpha_{2} N_{\vartheta}(s, t) \tilde{d}} z^{T}(s) z(s) d s\right\}
\end{aligned}
$$

The following fact is very obvious:

$$
\left(e^{\ln \left(\mu_{1} \mu_{2}+e^{\left|\alpha_{1}-\alpha_{2}\right| d_{\tau}}\right)}\right)^{N_{\vartheta}(s, t)} e^{\alpha_{1} T_{\mathrm{m}}(s, t)+\alpha_{2} T_{\mathrm{mism}}(s, t)} \geq e^{-\alpha_{1}(t+s)} .
$$


Then we can obtain

$$
\begin{aligned}
& \mathscr{E}\left\{\int_{0}^{t} e^{-\alpha_{1}(t+s)} \hat{r}^{T}(s) \hat{r}(s) d s\right\} \\
& \quad \leq \gamma_{0}^{2} \mathscr{E}\left\{\int_{0}^{t}\left(e^{\ln \left(\mu_{1} \mu_{2}+e^{\left|\alpha_{1}-\alpha_{2}\right| d \tau}\right)}\right)^{N_{\vartheta}(s, t)} e^{\alpha_{1}(t-s)+\alpha_{2} N_{\vartheta}(s, t)} \tilde{d} z^{T}(s) z(s) d s\right\} .
\end{aligned}
$$

Multiplying both side of (62) by

$$
e^{-N_{\vartheta}(0, t)\left(\ln \left(\mu_{1} \mu_{2}\right)+\left|\alpha_{1}-\alpha_{2}\right| d_{\tau}+\alpha_{2} \tilde{d}\right)}
$$

we can have

$$
\begin{aligned}
& \mathscr{E}\left\{\int_{0}^{t} e^{-N_{\vartheta}(0, t)\left(\ln \left(\mu_{1} \mu_{2}\right)+\left|\alpha_{1}-\alpha_{2}\right| d_{\tau}+\alpha_{2} \tilde{d}\right)} e^{-\alpha_{1}(t+s)} \hat{r}^{T}(s) \hat{r}(s) d s\right\} \\
& \quad \leq \gamma_{0}^{2} \mathscr{E}\left\{\int_{0}^{t} e^{-N_{\vartheta}(0, s)\left(\ln \left(\mu_{1} \mu_{2}\right)+\left|\alpha_{1}-\alpha_{2}\right| d_{\tau}+\alpha_{2} \tilde{d}\right)} e^{\alpha_{1}(t-s)} z^{T}(s) z(s) d s\right\} \\
& \quad \leq \gamma_{0}^{2} \mathscr{E}\left\{\int_{0}^{t} e^{\alpha_{1}(t-s)} z^{T}(s) z(s) d s\right\} .
\end{aligned}
$$

Let $N_{0}=0, t=T$, if (25) is satisfied, we can conclude that

$$
\mathscr{E}\left\{\int_{0}^{T} e^{-\alpha_{1}(2 t+s)} \hat{r}^{T}(s) \hat{r}(s) d s\right\} \leq \gamma_{0}^{2} e^{\alpha_{1} T} \mathscr{E}\left\{\int_{0}^{T} z^{T}(s) z(s) d s\right\}
$$

By simple calculation, we obtain

$$
\mathscr{E}\left\{\int_{0}^{T} e^{-\alpha_{1} s \hat{r}^{T}}(s) \hat{r}(s) d s\right\} \leq\left(\gamma_{0} e^{\frac{3 \alpha_{1} T}{2}}\right)^{2} \mathscr{E}\left\{\int_{0}^{T} z^{T}(s) z(s) d s\right\},
$$

where $\gamma=\gamma_{0} e^{\frac{3 \alpha_{1} T}{2}}, \lambda=\alpha_{1}$, which implies that the $H_{\infty}$ property (12) is guaranteed. This proof is completed.

In this section, we direct our attention to design a detector/controller unit in the form of (2) based on the results of Theorem 3.1 which guarantees the system (10) is SSFTS with $H_{\infty}$ performance index (12).

Remark 3.1 In the proof of the Theorem 3.1, we introduce some mode-dependent Lyapunov function (38)-(43) in match period, and the exponential structure such as $e^{\alpha_{1}(t-s)}$, not is $e^{-\alpha_{1}(t-s)}$. The reason why this is constructed, one is that the proof of SSFTS is more convenient, the other is that the restricted condition such as $P_{i} \leq \mu_{1} P_{i j}, P_{i j} \leq \mu_{2} P_{i}$ is not necessary. So, the results in this paper are more reasonable.

Theorem 3.2 For any $i, j \in \mathcal{K}, p, q \in \Delta_{1}, \alpha_{1}>0, \alpha_{2}>0$, matrices $\tilde{R}_{p q}$ and any real scalars $\rho_{i}$, the augmented system (10) is SSFTS with respect given $\left(c_{1}, c_{2}, \mathscr{G}, T\right)$ and the performance index (12) is satisfied, if there exist positive definite matrices $P_{i p q}, P_{w p q}, Q_{l p q}, l=1,2,3$, $\bar{R}_{1 p q}, \bar{R}_{2 p q}, W_{1 p q}, \bar{W}_{1 p q}, W_{21 p q}, W_{22 p q}$ symmetric matrices $\mathscr{H}_{1}, \mathscr{H}_{2}$ and any real matrices 
$T_{k}, k=1,2,3,4,5, \mathfrak{G}, S_{p q}, S_{\text {wpq }}, \mathscr{M}_{i p q}^{(11)}, \mathscr{M}_{i q}^{(22)}, \widehat{K}_{i q}, \widehat{A}_{i q}, \widehat{B}_{i q}, \widehat{C}_{r i q}, \widehat{D}_{\text {riq }}$, and positive scalar $\gamma_{0}$ such that (23)-(25) are satisfied and following LMIs hold:

$$
\begin{aligned}
& \Phi_{i p q}^{d_{1}}=\left[\begin{array}{ccccccccc}
\Phi_{11}^{(p q)} & \Phi_{12}^{(p q)} & \Theta_{13}^{(p q)} & 0 & \Phi_{15}^{(p q)} & 0 & \Phi_{17}^{(p q)} & \Phi_{18}^{(p q)} & \Phi_{19}^{(p q)} \\
* & \Phi_{22}^{(p q)} & \Theta_{23}^{(p q)} & \Theta_{24}^{(p q)} & 0 & 0 & \Theta_{27}^{(p q)} & \Phi_{28}^{(p q)} & 0 \\
* & * & \Theta_{33}^{(p q)} & \Theta_{34}^{(p q)} & 0 & 0 & \Theta_{37}^{(p q)} & \Theta_{38}^{(p q)} & 0 \\
* & * & * & \Theta_{44}^{(p q)} & 0 & 0 & \Theta_{47}^{(p q)} & \Theta_{48}^{(p q)} & 0 \\
* & * & * & * & \Phi_{55}^{(p q)} & 0 & 0 & \Phi_{58}^{(p q)} & 0 \\
* & * & * & * & * & \Theta_{66}^{(p q)} & \Theta_{67}^{(p q)} & \Theta_{68} & 0 \\
* & * & * & * & * & * & \Theta_{77}^{(p q)} & \Phi_{78}^{(p q)} & 0 \\
* & * & * & * & * & * & * & \Phi_{88}^{(p q)} & 0 \\
* & * & * & * & * & * & * & * & \Theta_{99}
\end{array}\right]<0, \\
& \Phi_{i p q}^{d_{2}}=\left[\begin{array}{ccccccccc}
\Phi_{11}^{(p q)} & \Phi_{12}^{(p q)} & \Theta_{13}^{(p q)} & 0 & \Phi_{15}^{(p q)} & 0 & \Phi_{17}^{(p q)} & \Phi_{18}^{(p q)} & \Phi_{19}^{(p q)} \\
* & \Phi_{22}^{(p q)} & \Theta_{23}^{(p q)} & \Theta_{24}^{(p q)} & 0 & 0 & \Theta_{27}^{(p q)} & \bar{\Phi}_{28}^{(p q)} & 0 \\
* & * & \Theta_{33}^{(p q)} & \Theta_{34}^{(p q)} & 0 & 0 & \Theta_{37}^{(p q)} & \bar{\Theta}_{38}^{(p q)} & 0 \\
* & * & * & \Theta_{44}^{(p q)} & 0 & 0 & \Theta_{47}^{(p q)} & \bar{\Theta}_{48}^{(p q)} & 0 \\
* & * & * & * & \Phi_{55}^{(p q)} & 0 & 0 & \Phi_{58}^{(p q)} & 0 \\
* & * & * & * & * & \Theta_{66}^{(p q)} & \Theta_{67}^{(p q)} & \Theta_{68} & 0 \\
* & * & * & * & * & * & \Theta_{77}^{(p q)} & \bar{\Phi}_{78}^{(p q)} & 0 \\
* & * & * & * & * & * & * & \Phi_{88}^{(p q)} & 0 \\
* & * & * & * & * & * & * & * & \Theta_{99}
\end{array}\right]<0, \\
& \Phi_{i p}^{d_{1}}=\left[\begin{array}{ccccccccc}
\Phi_{11}^{(p)} & \Phi_{12}^{(p)} & \Theta_{13}^{(p)} & 0 & \Phi_{15}^{(p)} & 0 & \Phi_{17}^{(p)} & \Phi_{18}^{(p)} & \Phi_{19}^{(p)} \\
* & \Phi_{22}^{(p)} & \Theta_{23}^{(p)} & \Theta_{24}^{(p)} & 0 & 0 & \Theta_{27}^{(p)} & \Phi_{28}^{(p)} & 0 \\
* & * & \Theta_{33}^{(p)} & \Theta_{34}^{(p)} & 0 & 0 & \Theta_{37}^{(p)} & \Theta_{38}^{(p)} & 0 \\
* & * & * & \Theta_{44}^{(p)} & 0 & 0 & \Theta_{47}^{(p)} & \Theta_{48}^{(p)} & 0 \\
* & * & * & * & \Phi_{55}^{(p)} & 0 & 0 & \Phi_{58}^{(p)} & 0 \\
* & * & * & * & * & \Theta_{66}^{(p)} & \Theta_{67}^{(p)} & \Theta_{68} & 0 \\
* & * & * & * & * & * & \Theta_{77}^{(p)} & \Phi_{78}^{(p)} & 0 \\
* & * & * & * & * & * & * & \Phi_{88}^{(p)} & 0 \\
* & * & * & * & * & * & * & * & \Theta_{99}
\end{array}\right]<0, \\
& \Phi_{i p}^{d_{2}}=\left[\begin{array}{ccccccccc}
\Phi_{11}^{(p)} & \Phi_{12}^{(p)} & \Theta_{13}^{(p)} & 0 & \Phi_{15}^{(p)} & 0 & \Phi_{17}^{(p)} & \Phi_{18}^{(p)} & \Phi_{19}^{(p)} \\
* & \Phi_{22}^{(p)} & \Theta_{23}^{(p)} & \Theta_{24}^{(p)} & 0 & 0 & \Theta_{27}^{(p)} & \bar{\Phi}_{28}^{(p)} & 0 \\
* & * & \Theta_{33}^{(p)} & \Theta_{34}^{(p)} & 0 & 0 & \Theta_{37}^{(p)} & \bar{\Theta}_{38}^{(p)} & 0 \\
* & * & * & \Theta_{44}^{(p)} & 0 & 0 & \Theta_{47}^{(p)} & \bar{\Theta}_{48}^{(p)} & 0 \\
* & * & * & * & \Phi_{55}^{(p)} & 0 & 0 & \Phi_{58}^{(p)} & 0 \\
* & * & * & * & * & \Theta_{66}^{(p)} & \Theta_{67}^{(p)} & \Theta_{68} & 0 \\
* & * & * & * & * & * & \Theta_{77}^{(p)} & \bar{\Phi}_{78}^{(p)} & 0 \\
* & * & * & * & * & * & * & \Phi_{88}^{(p)} & 0 \\
* & * & * & * & * & * & * & * & \Theta_{99}
\end{array}\right]<0,
\end{aligned}
$$


where

$$
\begin{aligned}
& \Phi_{11}^{(p q)}=\left[\begin{array}{cc}
H e\left(\mathscr{M}_{i p q}^{(11)} A_{i p}\right) & \bar{T}_{i p}^{T}\left[\begin{array}{c}
\widehat{K}_{i q} \\
0
\end{array}\right]+C_{1 p}^{T} \widehat{B}_{i q}^{T} \\
* & H e\left(\widehat{A}_{i q}\right)+\rho_{i} \Phi_{2 i}
\end{array}\right]-\tilde{E}_{p q}^{T}\left[\begin{array}{cc}
-\alpha_{2} P_{i p q}^{(11)} & 0 \\
0 & -\alpha_{2} P_{i p q}^{(22)}
\end{array}\right] E_{p q} \\
& +\tilde{E}_{p q}^{T}\left[\begin{array}{cc}
\sum_{j=1, j \neq i}^{\tilde{k}} \bar{\pi}_{i j}^{(p q)}\left(P_{j p q}^{(11)}-P_{i p q}^{(11)}\right)+\bar{d}^{2} W_{21 p q}^{(11)} & 0 \\
0 & \sum_{j=1, j i i}^{\tilde{k}} \bar{\pi}_{i j}^{(p q)}\left(P_{j p q}^{(22)}-P_{i p q}^{(22)}\right)+\bar{d}^{2} W_{21 p q}^{(22)}
\end{array}\right] E_{p q} \\
& +Q_{1 p q}+Q_{2 p q}+Q_{3 p q}+\tau_{1}^{2} \bar{R}_{1 p q}+\bar{\tau}^{2} \bar{R}_{2 p q}+H e\left(T_{51} E_{p q}\right)+\sum_{j=1, j \neq i}^{\tilde{k}} \frac{1}{4}\left(\delta_{i j}^{(p q)}\right)^{2} \varepsilon_{i j},
\end{aligned}
$$

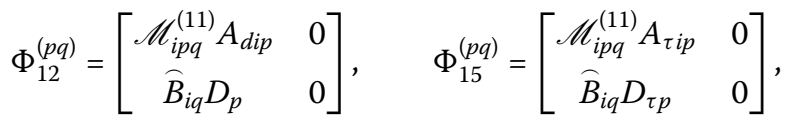

$$
\begin{aligned}
& \Phi_{17}^{(p q)}=\left[0\left[\begin{array}{c}
\bar{T}_{i p}^{T}\left[\begin{array}{c}
\hat{K}_{i q} \\
0
\end{array}\right] \\
0
\end{array}\right]\left[\begin{array}{cc}
\mathscr{M}_{i p q}^{(11)} B_{h i p} & \mathscr{M}_{i p q}^{(11)} B_{f i p} \\
\widehat{B}_{i q} D_{h p} & \widehat{B}_{i q} D_{f p}
\end{array}\right]\right] \text {, }
\end{aligned}
$$

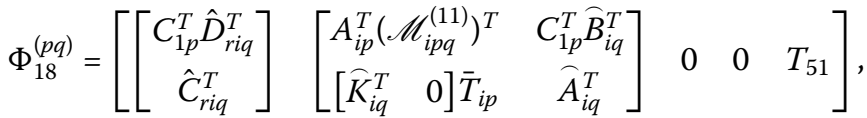

$$
\begin{aligned}
& \Phi_{19}^{(p q)}=\left[\tilde{E}_{p q}^{T}\left[\begin{array}{cc}
P_{1 p q}^{(11)}-P_{i p q}^{(11)} & 0 \\
0 & P_{1 p q}^{(22)}-P_{i p q}^{(22)}
\end{array}\right] \tilde{E}_{p q} \cdots\right. \\
& \tilde{E}_{p q}^{T}\left[\begin{array}{cc}
P_{(i-1) p q}^{(11)}-P_{i p q}^{(11)} & 0 \\
0 & P_{(i-1) p q}^{(22)}-P_{i p q}^{(22)}
\end{array}\right] \tilde{E}_{p q} \\
& \tilde{E}_{p q}^{T}\left[\begin{array}{cc}
P_{(i+1) p q}^{(11)}-P_{i p q}^{(11)} & 0 \\
0 & P_{(i+1) p q}^{(22)}-P_{i p q}^{(22)}
\end{array}\right] \tilde{E}_{p q} \cdots \\
& \left.\tilde{E}_{p q}^{T}\left[\begin{array}{cc}
P_{\tilde{k} p q}^{(11)}-P_{i p q}^{(11)} & 0 \\
0 & P_{\tilde{k} p q}^{(22)}-P_{i p q}^{(22)}
\end{array}\right] \tilde{E}_{p q}\right], \\
& \Phi_{22}^{(p q)}=\tilde{E}_{p q}^{T}\left[\begin{array}{cc}
\bar{d} \mathscr{H}_{1}^{(11)}-\bar{d} \mathscr{H}_{2}^{(11)}-H e\left(\mathfrak{G}_{2}^{(11)}\right)+2 W_{22 p q} & \bar{d} \mathscr{H}_{1}^{(11)}-\bar{d} \mathscr{H}_{2}^{(11)}-H e\left(\mathfrak{G}_{2}^{(11)}\right)+2 W_{22 p q}
\end{array}\right] \tilde{E}_{p q} \\
& -(1-d) e^{-\alpha_{2} d_{2}} Q_{1 p q}+H e\left(\left(T_{12}+T_{22}-T_{32}+T_{42}\right) \tilde{E}_{p q}\right) \text {, }
\end{aligned}
$$

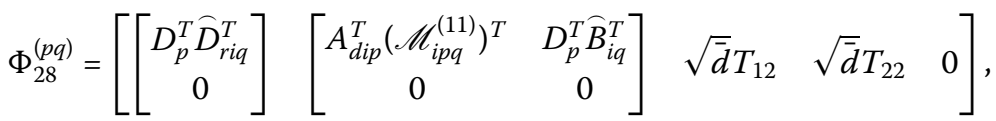

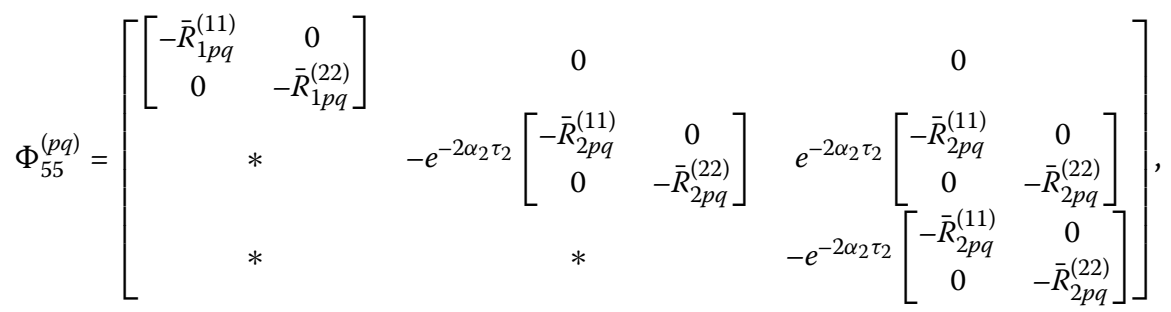

$$
\begin{aligned}
& \Phi_{58}^{(p q)}=\left[\left[\left[\begin{array}{c}
D_{\tau p}^{T} \widehat{D}_{r i q}^{T} \\
0
\end{array}\right]\left[\begin{array}{cc}
A_{\tau i p}^{T}\left(M_{i p q}^{(11)}\right)^{T} & D_{\tau p}^{T} \widehat{B}_{i q}^{T} \\
0 & 0
\end{array}\right] 0_{n \times 3 n}\right]\right. \text {, }
\end{aligned}
$$




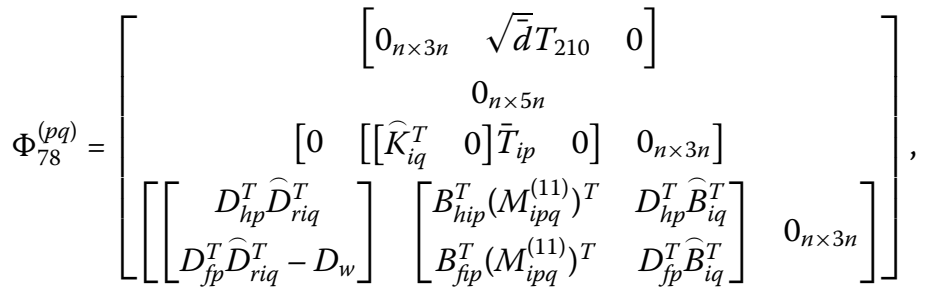

$$
\begin{aligned}
& \Phi_{88}^{(p q)}=\operatorname{diag}\left(-I \quad \Delta-H e\left(M_{i p q}\right) \quad-W_{1} \quad-3 W_{1} \quad-\varepsilon^{-1} \bar{W}_{1}\right),
\end{aligned}
$$

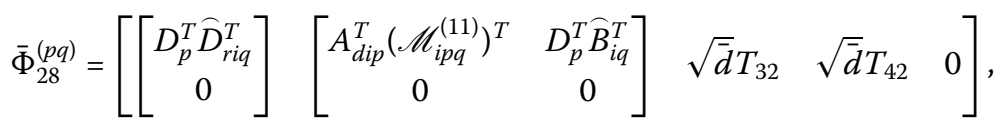

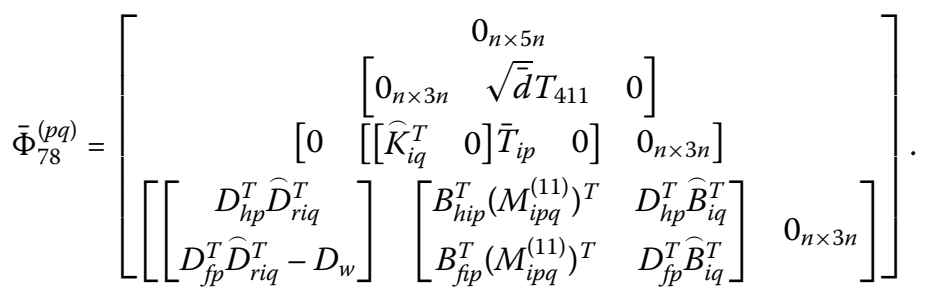

If we replace the switching signal $\tilde{\vartheta}_{t}=q$ and positive scalar $\alpha_{2}$ with switching signal $\vartheta_{t}=p$, and positive scalar $\alpha_{1}$ in the LMIs (66) and (67), we can obtain the LMIs (68) and (69), respectively.

Also, $\forall t \in\left[t_{k}, t_{k}+d_{k}\right)$, the detector/controller unit gain matrices are given by

$$
\begin{aligned}
& \hat{A}_{i q}=\left(\mathscr{M}_{i q}^{(22)}\right)^{-1} \widehat{A}_{i q}, \quad \hat{B}_{i q}=\left(\mathscr{M}_{i q}^{(22)}\right)^{-1} \widehat{B}_{i q}, \quad \hat{C}_{r i q}=\widehat{C}_{r i q}, \\
& \hat{D}_{r i q}=\widehat{D}_{r i q}, \quad \hat{K}_{i q}=\left(\mathfrak{M}_{i q}^{(11)}\right)^{-1} \widehat{K}_{i q}
\end{aligned}
$$

$\forall t \in\left[t_{k}+d_{k}, t_{k+1}\right)$, the detector/controller unit gain matrices are given by

$$
\begin{aligned}
& \hat{A}_{i p}=\left(\mathscr{M}_{i p}^{(22)}\right)^{-1} \widehat{A}_{i p}, \quad \hat{B}_{i p}=\left(\mathscr{M}_{i p}^{(22)}\right)^{-1} \widehat{B}_{i p}, \quad \hat{C}_{r i p}=\widehat{C}_{r i p}, \\
& \hat{D}_{\text {rip }}=\widehat{D}_{\text {rip }}, \quad \hat{K}_{i p}=\left(\mathfrak{M}_{i p}^{(11)}\right)^{-1} \widehat{K}_{i p} .
\end{aligned}
$$

Proof From Theorem 3.1 we know that if LMIs (19) and (20) are satisfied in mismatch period, then inequality (37) holds, that is, $\forall t \in\left[t_{k}, t_{k}+d_{k}\right.$ ), system (10) is SSFTS and the $H_{\infty}$ property (12) will be guaranteed under the conditions (19) and (20). In order to obtain proper detector/controller unit gain matrices, we should decompose the matrices $\mathscr{M}_{i p q}$ as follows:

$$
\mathscr{M}_{i p q}=\left[\begin{array}{cc}
\mathscr{M}_{i p q}^{(11)} & 0 \\
0 & \mathscr{M}_{i q}^{(22)}
\end{array}\right],
$$

and based on Assumption 2.1, we let

$$
\mathscr{M}_{i p q}^{(11)}=\bar{T}_{i p}^{T}\left[\begin{array}{cc}
\mathfrak{M}_{i q}^{(11)} & 0 \\
0 & \mathfrak{M}_{i q}^{(22)}
\end{array}\right] \bar{T}_{i p} .
$$


Then,

$$
\begin{aligned}
& {\left[\begin{array}{cc}
\mathscr{M}_{i p q}^{(11)} & 0 \\
0 & \mathscr{M}_{i q}^{(22)}
\end{array}\right]\left[\begin{array}{cc}
A_{i p} & B_{i p} \hat{K}_{i q} \\
\hat{B}_{i q} C_{1 p} & \hat{A}_{i q}
\end{array}\right]=\left[\begin{array}{cc}
\mathscr{M}_{i p q}^{(11)} A_{i p} & \mathscr{M}_{i p q}^{(11)} B_{i p} \hat{K}_{i q} \\
\mathscr{M}_{i q}^{(22)} \hat{B}_{i q} C_{1 p} & \mathscr{M}_{i q}^{(22)} \hat{A}_{i q}
\end{array}\right],} \\
& \mathscr{M}_{i p q}^{(11)} B_{i p} \hat{K}_{i q}=\bar{T}_{i p}^{T}\left[\begin{array}{cc}
\mathfrak{M}_{i q}^{(11)} & 0 \\
0 & \mathfrak{M}_{i q}^{(22)}
\end{array}\right] \bar{T}_{i p} B_{i p} \hat{K}_{i q}=\bar{T}_{i p}^{T}\left[\begin{array}{c}
\mathfrak{M}_{i q}^{(11)} \hat{K}_{i q} \\
0
\end{array}\right] .
\end{aligned}
$$

Now, letting $\widehat{A}_{i q}=\mathscr{M}_{i q}^{(22)} \hat{A}_{i q}, \widehat{B}_{i q}=\mathscr{M}_{i q}^{(22)} \hat{B}_{i q}, \widehat{C}_{r i q}=\hat{C}_{r i q}, \widehat{D}_{r i q}=\hat{D}_{r i q}, \widehat{K}_{i q}=\mathfrak{M}_{i q}^{(11)} \hat{K}_{i q}$, then we substitute (72)-(74) into (19) and (20), we know that (66), (67) are equivalent to (19), (20), respectively, furthermore, similar to the above discussion, we can easily derive that in match period LMIs (68), (69) are equivalent to (21), (22) as is obvious. This proof is completed.

Remark 3.2 In this paper, we select most variable matrices as diagonal matrices, for example: any matrix $T_{12}=\left[\begin{array}{cc}T_{12}^{(11)} & 0 \\ 0 & T_{12}^{(22)}\end{array}\right]$, the construction of the other matrices is similar to this structure, and such processing may affect the system. But this selection will greatly reduce the complexity of computing and cost of the system implementation.

Theorem 3.3 Under any switch signal, the augmented system (10) with dwell time constraint (25) is SSFTS and also satisfies $H_{\infty}$ property (12), if LMI conditions (19)-(25) and (66)-(69) hold. Moreover, matrices $\hat{A}_{i q}, \hat{B}_{i q}, \hat{K}_{i q}, \hat{C}_{\text {riq }}$ and $\hat{D}_{\text {riq }}$ can be obtained from (70) and (71), respectively. Further, based on Theorem 3.1 and Theorem 3.2, the simultaneous finite-time control and fault detection problem is converted into the following optimization: for given positive constant weighs $\varpi$,

$$
\begin{aligned}
& \min \bar{\gamma}=\varpi \gamma_{0}^{2} \\
& \text { s.t. (19)-(25), (66)-(69). }
\end{aligned}
$$

The next step is to evaluate the residual signal and compare it with some threshold values to detect the fault in the system.

In this paper, the residual evaluation function based on the root mean square energy of the residual signal is used.

$$
J_{\hat{r}}(t)=\sqrt{\frac{1}{T} \int_{0}^{T} \hat{r}^{T}(s) \hat{r}(s) d s}
$$

and the threshold $J_{t h}$ is obtained by

$$
J_{t h}=\sup _{\substack{f(t)=0 \\ h(t) \in L_{2}}} J_{\hat{r}}(t)
$$

therefore, the controller/detector unit is obtained such that the performance (12) holds and the constant threshold value $J_{t h}$ can be defined as $J_{t h}=\frac{\gamma\|h(t)\|_{2}}{\sqrt{T}}$. Finally the occurrence 
of fault can be detected by the following logic rule:

$$
\begin{aligned}
& J_{\hat{r}}(t)>J_{t h} \Rightarrow \text { alarm, } \\
& J_{\hat{r}}(t) \leq J_{t h} \Rightarrow \text { no faults. }
\end{aligned}
$$

\section{Examples}

In this section, we shall give an illustrative example to demonstrate the effectiveness of the proposed method. Firstly we consider the transition rate matrix $\Pi^{(p q)}$ and singular matrix $E_{p}$ with two vertices:

$$
\begin{array}{ll}
\Pi^{(1)}=\left[\begin{array}{cc}
-0.6+\Delta \bar{\pi}_{11}^{(1)} & 0.6+\Delta \bar{\pi}_{12}^{(1)} \\
0.8+\Delta \bar{\pi}_{21}^{(1)} & -0.8+\Delta \bar{\pi}_{22}^{(1)}
\end{array}\right], & \Pi^{(12)}=\left[\begin{array}{cc}
-0.6+\Delta \bar{\pi}_{11}^{(12)} & 0.6+\Delta \bar{\pi}_{12}^{(12)} \\
0.8+\Delta \bar{\pi}_{21}^{(12)} & -0.8+\Delta \bar{\pi}_{22}^{(12)}
\end{array}\right], \\
\Pi^{(2)}=\left[\begin{array}{cc}
-1.3+\Delta \bar{\pi}_{11}^{(2)} & 1.3+\Delta \bar{\pi}_{12}^{(2)} \\
1.5+\Delta \bar{\pi}_{21}^{(2)} & -1.5+\Delta \bar{\pi}_{22}^{(2)}
\end{array}\right], & \Pi^{(21)}=\left[\begin{array}{cc}
-1.3+\Delta \bar{\pi}_{11}^{(21)} & 1.3+\Delta \bar{\pi}_{12}^{(21)} \\
1.5+\Delta \bar{\pi}_{21}^{(21)} & 1.5+\Delta \bar{\pi}_{22}^{(21)}
\end{array}\right], \\
E_{1}=E_{2}=\left[\begin{array}{ll}
1 & 0 \\
0 & 0
\end{array}\right], & R_{1}=R_{2}=\left[\begin{array}{l}
0 \\
1
\end{array}\right],
\end{array}
$$

where $\delta_{11}^{(1)}=\delta_{12}^{(1)}=\delta_{11}^{(12)}=\delta_{12}^{(12)}=0.1, \delta_{21}^{(1)}=\delta_{22}^{(1)}=\delta_{21}^{(12)}=\delta_{22}^{(12)}=0.2, \delta_{11}^{(2)}=\delta_{12}^{(2)}=\delta_{11}^{(21)}=\delta_{12}^{(21)}=$ $0.15, \delta_{21}^{(2)}=\delta_{22}^{(2)}=\delta_{21}^{(21)}=\delta_{22}^{(21)}=0.3$.

Secondly, we introduce the following parameters into the augmented system (10):

$$
\begin{aligned}
& A_{11}=\left[\begin{array}{cc}
0.08 & -0.15 \\
3 & -0.72
\end{array}\right], \quad A_{12}=\left[\begin{array}{cc}
0.079 & -0.15 \\
1 & -0.721
\end{array}\right], \\
& A_{21}=\left[\begin{array}{cc}
-0.08 & -1.25 \\
0.22 & -0.20
\end{array}\right], \quad A_{22}=\left[\begin{array}{cc}
1.08 & -0.14 \\
0.15 & -1.32
\end{array}\right], \\
& A_{d 11}=\left[\begin{array}{cc}
-0.02 & -1.15 \\
0.23 & 0.152
\end{array}\right], \quad A_{d 12}=\left[\begin{array}{cc}
-0.13 & 0.29 \\
0.108 & -0.135
\end{array}\right], \\
& A_{d 21}=\left[\begin{array}{cc}
0.38 & 1.15 \\
0.022 & -0.12
\end{array}\right], \quad A_{d 22}=\left[\begin{array}{cc}
-1.8 & 0.14 \\
0.15 & -0.3
\end{array}\right], \\
& A_{\tau 11}=\left[\begin{array}{cc}
1.12 & -1.51 \\
0.13 & 0.002
\end{array}\right], \quad A_{\tau 12}=\left[\begin{array}{cc}
0.103 & 1.19 \\
0.108 & 0.035
\end{array}\right], \\
& A_{\tau 21}=\left[\begin{array}{cc}
0.128 & 0.105 \\
1.122 & -0.22
\end{array}\right], \quad A_{\tau 22}=\left[\begin{array}{cc}
-0.08 & 0.124 \\
0.55 & -0.13
\end{array}\right], \\
& B_{11}=B_{12}=B_{21}=B_{22}=\left[\begin{array}{c}
-0.1 \\
0.5
\end{array}\right], \quad B_{h 11}=B_{h 21}=\left[\begin{array}{l}
0.1 \\
0.1
\end{array}\right], \\
& B_{h 12}=B_{h 22}=\left[\begin{array}{c}
0.2 \\
-0.1
\end{array}\right], \quad B_{f 11}=B_{f 21}=\left[\begin{array}{cc}
0.01 \\
0.12
\end{array}\right], \\
& B_{f 12}=B_{f 22}=\left[\begin{array}{l}
0.25 \\
0.32
\end{array}\right], \quad C_{11}=C_{12}=\left[\begin{array}{cc}
0.2 & 0 \\
0 & 0.1
\end{array}\right],
\end{aligned}
$$




$$
\begin{aligned}
& D_{11}=D_{12}=\left[\begin{array}{cc}
0.3 & 0 \\
0 & 0.5
\end{array}\right], \quad D_{\tau 1}=D_{\tau 2}=\left[\begin{array}{cc}
0.15 & 1.2 \\
0 & 0.05
\end{array}\right], \\
& D_{h 1}=D_{h 2}=D_{f 1}=D_{f 2}=\left[\begin{array}{c}
0 \\
1.2
\end{array}\right],
\end{aligned}
$$

The systems matrices of model (11) are chosen as

$$
A_{w}=\left[\begin{array}{cc}
0.1 & 0 \\
0 & 0.2
\end{array}\right], \quad B_{w}=\left[\begin{array}{l}
1 \\
0
\end{array}\right], \quad C_{w}=\left[\begin{array}{ll}
2 & 0
\end{array}\right], \quad D_{w}=0.1
$$

We assume that error tolerance $\rho_{1}=0.5, \rho_{2}=0.8$, lag time $d_{k}$ satisfies $0 \leq d_{k} \leq 1$ and let $\tilde{d}=0.8$. Time-varying delay $d(t)=\tau(t)=0.1+0.1 \sin (10 t)$, then we can obtain $d_{1}=\tau_{1}=0.1$, $d_{2}=\tau_{2}=0.2$ very easily. Letting $c_{1}=1, c_{2}=5, T=30, \alpha_{1}=0.1, \alpha_{2}=0.2$,

$$
\bar{T}_{11}=\bar{T}_{12}=\bar{T}_{21}=\bar{T}_{22}=\left[\begin{array}{cc}
-0.3845 & 1.9231 \\
0.3590 & 0.0718 \\
1.2875 & 0.2575
\end{array}\right]
$$

and using Theorem 3.2, the controller/detectors unit are obtained as follows:

$$
\begin{aligned}
& {\left[\begin{array}{lll}
\hat{A}_{11} & \hat{B}_{11} & \hat{K}_{11}
\end{array}\right]} \\
& =\left[\begin{array}{cccccc}
-1.0101 & 0.0011 & -0.0161 & -0.0220 & & \\
0.0081 & -1.3421 & 0.0183 & 0.0556 & -0.0908 & -0.0321 \\
\hline-0.0102 & -0.0419 & -1.7878 & 0.5138 & -1.6061 & -0.1901
\end{array}\right], \\
& {\left[\begin{array}{lll}
\hat{A}_{12} & \hat{B}_{12} & \hat{K}_{12}
\end{array}\right]} \\
& =\left[\begin{array}{cccccc}
-1.3019 & 0.0166 & -0.0218 & -0.0401 & & \\
0.0901 & -1.3131 & 0.0011 & 0.0291 & -0.0528 & -0.0012 \\
\hline-0.0510 & -0.0305 & -1.0011 & 0.8102 & -1.0115 & -0.0529
\end{array}\right], \\
& {\left[\begin{array}{lll}
\hat{A}_{21} & \hat{B}_{21} & \hat{K}_{21}
\end{array}\right]} \\
& =\left[\begin{array}{cccccc}
-1.0129 & 0.0428 & -0.0484 & -0.0215 & & \\
0.0802 & -1.1002 & 0.0017 & 0.0106 & -0.0254 & -0.0075 \\
\hline-0.0119 & -0.0204 & -1.0702 & 0.3531 & -1.0820 & -0.0607
\end{array}\right], \\
& {\left[\begin{array}{lll}
\hat{A}_{22} & \hat{B}_{22} & \hat{K}_{22} \\
\hat{C}_{r 22} & \hat{D}_{r 22} &
\end{array}\right]} \\
& =\left[\begin{array}{cccccc}
-1.5298 & 0.0198 & -0.0152 & -0.0232 & & \\
0.0466 & -1.2045 & 0.0110 & 0.0144 & -0.0261 & -0.0830 \\
\hline-0.0127 & -0.0405 & -1.0651 & 0.2518 & -1.0449 & -0.0942
\end{array}\right] \text {. }
\end{aligned}
$$

For the simulation purpose, we set

$$
f(t)= \begin{cases}1, & 10 \leq t \leq 17 \\ 0, & \text { otherwise }\end{cases}
$$




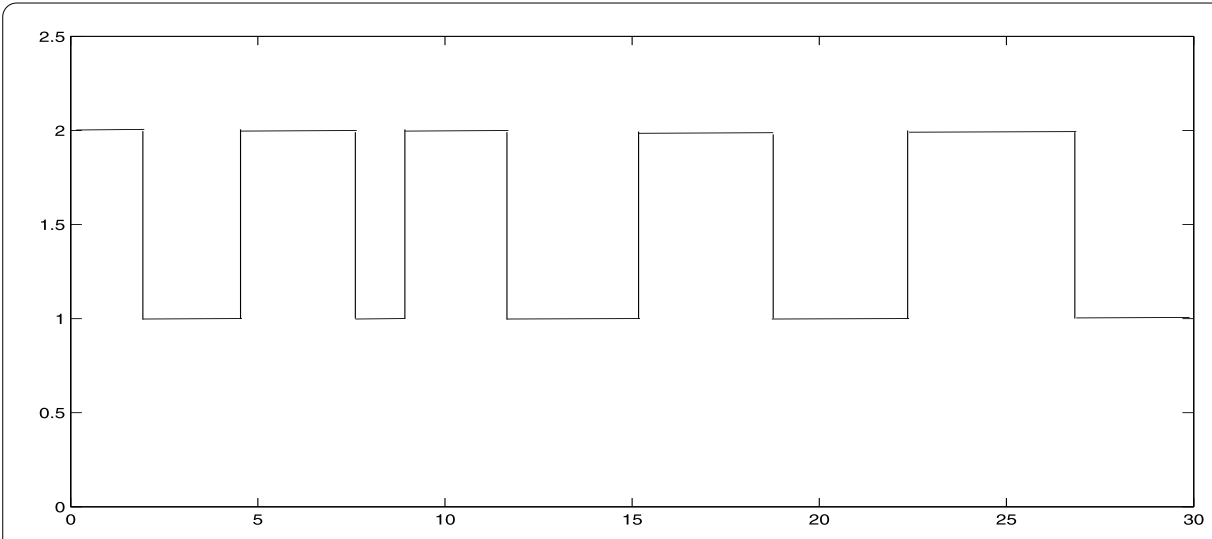

Figure 2 System jumping mode $v_{t}$

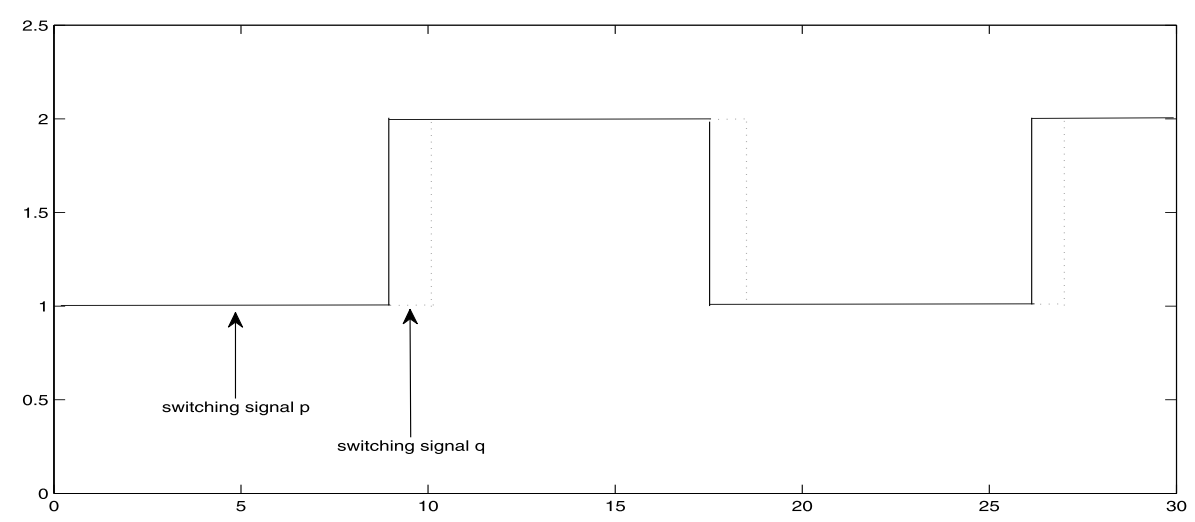

Figure 3 Switching signals $\vartheta_{t}$ and $\tilde{\vartheta}_{t}$

The initial modes are takes as $v_{0}=2, \vartheta_{0}=1$, respective. The simulation time is taken as 30 time units, and each unit length is taken as $t=5 \mathrm{~s}$. The jumping modes path from time step 0 to time step 30, the switching modes path is chosen according to the ADT $\tau_{a}>8.7091$ constraint, which are shown in Figs. 2 and 3, respectively. Further, the residual evaluation function are shown in Figs. 4 and 5, which means the fault is detected. From Fig. 4, we know that if we enlarge the parameters $D_{f 1}, D_{f 2}$, the effect of fault on the output $y(t)$ will be larger, so the residual signal $\hat{r}(t)$ becomes larger, and the detection time of fault will be reduced. Based on our results, we can obtain $J_{t h}=\sup _{h(t) \neq 0 \neq 0} \mathscr{E}\left(\frac{1}{30} \int_{0}^{30} \hat{r}^{T}(t) \hat{r}(t) d t\right)=0.1220$ and Fig. 5 show us that $\left(\frac{1}{10.02} \int_{0}^{10.02} \hat{r}^{T}(t) \hat{r}(t) d t\right)=0.2037>J_{t h}$, that is fault signal will be detected after $0.02 \mathrm{~s}$. Meantime, Fig. 6 shows the inter-event intervals, obviously, from Fig. 6, we can know that the event is triggered 145 times during the simulation time period. Finally, the phase plane plot of the closed-loop system is shown in Figs. 7 and 8 depicts that the states of the system (10) is stability in finite time with our proposed control strategy in this paper.

\section{Conclusions}

In this paper, the problem of simultaneous finite-time event-triggered control and fault detection for a class of singular Markovian mixed delay jump systems under asynchronous 
Loo et al. Advances in Difference Equations <wide>(2018<wide>) 2018:80

Page 29 of 32

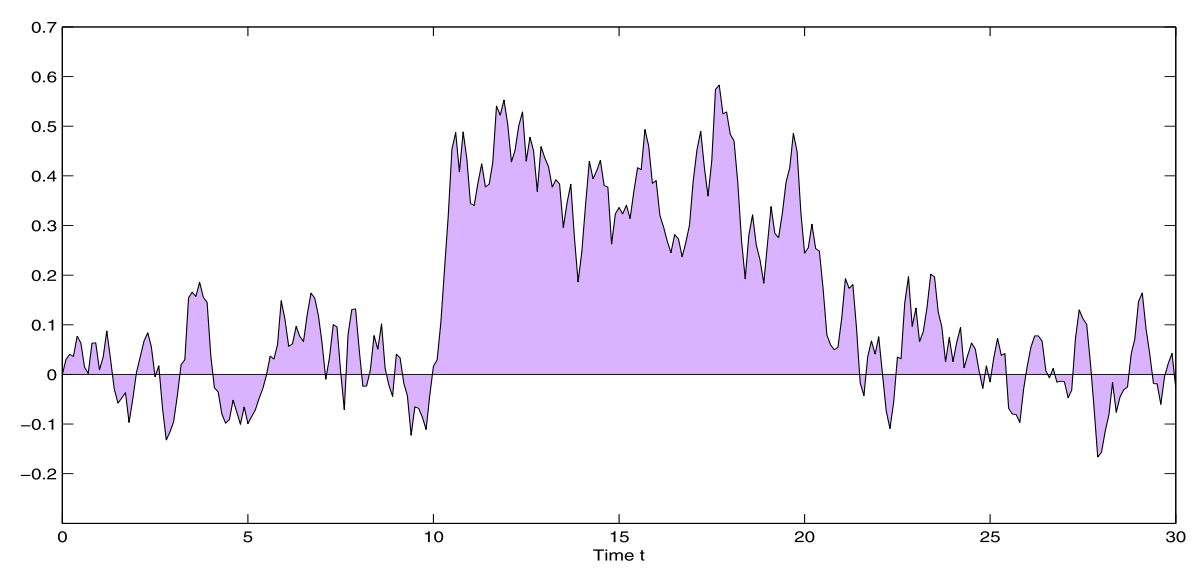

Figure 4 Residual signal $\hat{r}(t)$

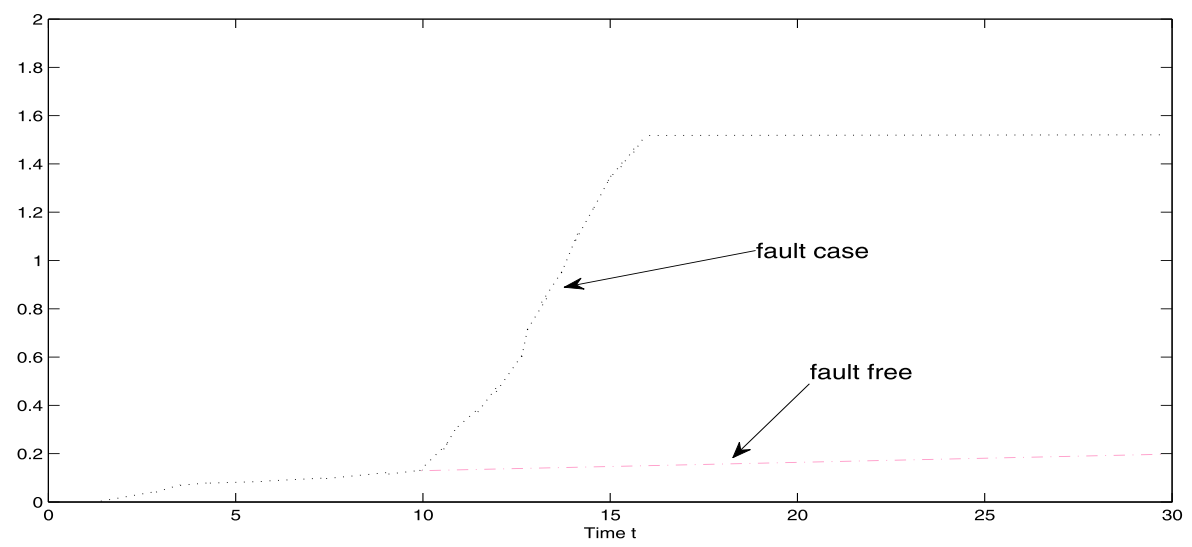

Figure 5 Residual evaluation function

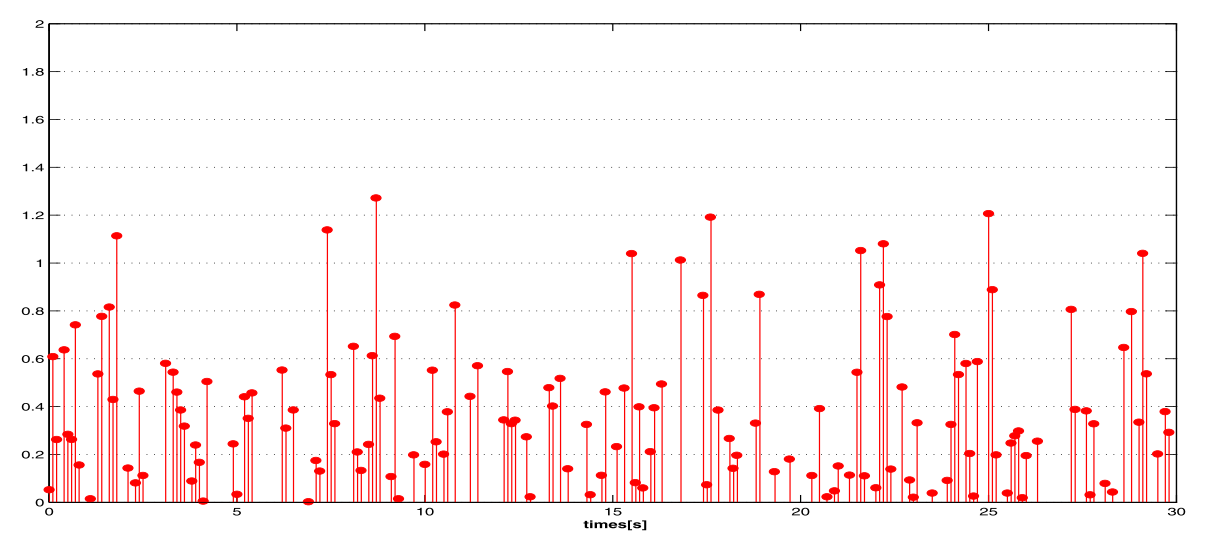

Figure 6 Inter-event intervals 


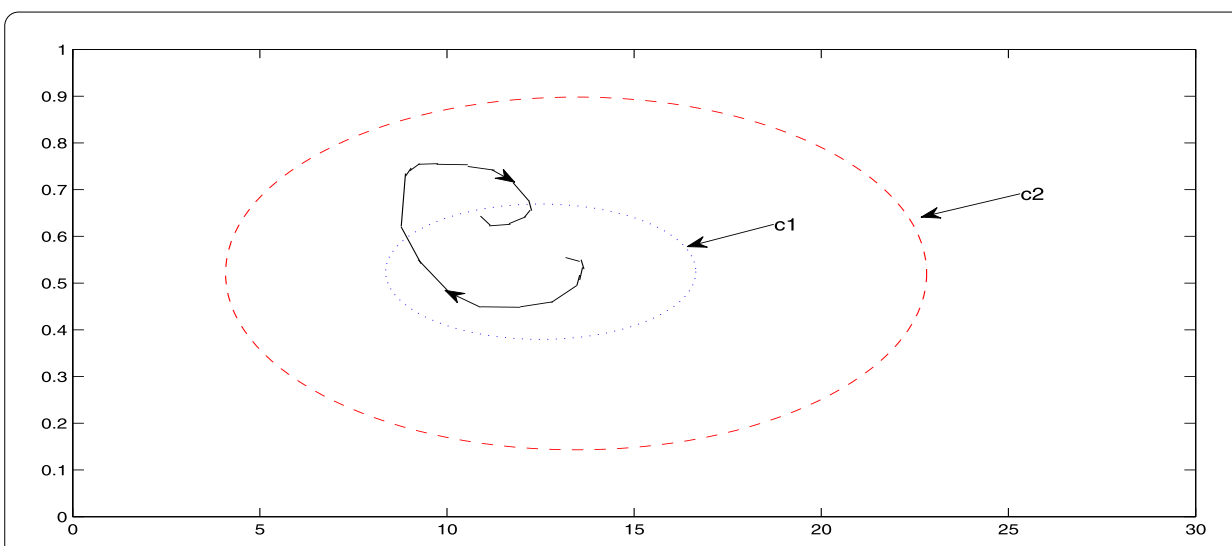

Figure 7 Phase plane plot of closed-loop system

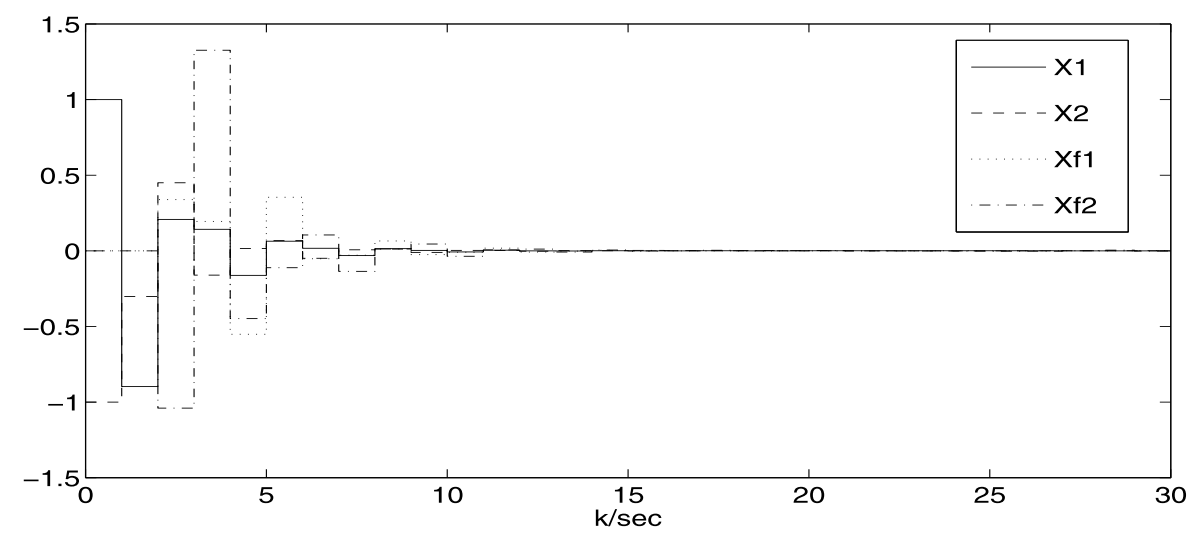

Figure 8 System responses with $u_{t}$

switching has been investigated. A mode-dependent detector/controller are designed, which guarantees the closed-loop system is SSFTS and satisfies four $H_{\infty}$ performance indices. By using some novel integral inequalities and the optimization technique, the results are derived in terms of the LMIs. Finally, a numerical example is provided to illustrate the effectiveness of the proposed method.

\section{Acknowledgements}

All authors are grateful to the anonymous referees for several comments and suggestions of improvement. This work was supported in part by the National Natural Science Foundation of China under Grants 11661028, 11661030, 11502057, the Natural Science Foundation of Guangxi under Grant 2015GXNSFBA139005, 2014GXNSFBA1 18023. This work was also supported by the China Scholarship Council (201608455012).

\section{Competing interests}

All the authors declare that they have no competing interests.

\section{Authors' contributions}

All three authors contributed equally to this work. They all read and approved the final version of the manuscript.

\section{Author details}

${ }^{1}$ College of Science, Guilin University of Technology, Guilin, P.R. China. ${ }^{2}$ School of Mathematical Sciences, University of Electronic Science and Technology of China, Chengdu, P.R. China. ${ }^{3}$ School of Science, Hubei University for Nationalities, Enshi, P.R. China. 


\section{Publisher's Note}

Springer Nature remains neutral with regard to jurisdictional claims in published maps and institutional affiliations.

\section{Received: 17 November 2017 Accepted: 20 February 2018 Published online: 05 March 2018}

\section{References}

1. Gao, H., Chen, T., Wang, L.: Robust fault detection with missing measurements. Int. J. Control 81, 804-819 (2008)

2. Luo, M., Zhong, S.: Robust fault detection of uncertain time-delay Markovian jump systems with different system modes. Circuits Syst. Signal Process. 33, 115-139 (2014)

3. Liu, J., Yue, D.: Event-triggering in networked systems with probabilistic sensor and actuator faults. Inf. Sci. 240, 145-160 (2013)

4. Dong, H., Wang, Z., Gao, H.: Fault detection for Markovian jump systems with sensor saturations and randomly varying nonlinearities. IEEE Trans. Circuits Syst. I, Regul. Pap. 59, 2354-2362 (2012)

5. Hwang, I., Kim, S., Kim, Y., Seah, C.E.: A survey of fault detection, isolation, and reconfiguration methods. IEEE Trans. Control Syst. Technol. 18, 636-653 (2010)

6. Jiang, B., Staroswiecki, M., Cocquempot, V.: $H_{\infty}$ fault detection filter design for linear discrete-time systems with multiple time delays. Int. J. Syst. Sci. 34, 365-373 (2003)

7. Wan, X., Fang, H.: Fault detection for discrete-time networked nonlinear systems with incomplete measurements. Int. J. Syst. Sci. 44, 2068-2081 (2013)

8. Shokouhi-Nejad, H., Rikhtehgar Ghiasi, A., Badamchizadeh, M.A.: Robust simultaneous finite-time control and fault detection for uncertain linear switched systems with time-varying delay. IET Control Theory Appl. 11, 1041-1052 (2017)

9. Zhong, G., Yang, G.: Robust control and fault detection for continuous-time switched systems subject to a dwell constraint. Int. J. Robust Nonlinear Control 25, 3799-3817 (2015)

10. Zhai, D., Lu, A., Li, J., Zhang, Q.: Simultaneous fault detection and control for switched linear systems with mode-dependent average dwell-time. Appl. Math. Comput. 272, 767-792 (2016)

11. Li, J., Park, J.H., Ye, D.: Simultaneous fault detection and control design for switched systems with two quantized signals. ISA Trans. 66, 296-309 (2017)

12. Shokouhi-Nejad, H., Rikhtehara Ghiasi, A., Badamchizadeh, M.A.: Robust simultaneous fault detection and control for class of nonlinear stochastic switched delay systems under asynchronous switching. J. Franklin Inst. (2017). https://doi.org/10.1016/j.jranklin.2017.05.037

13. Wang, H., Yang, G.: Simultaneous fault detection and control for uncertain linear discrete-time systems. IET Control Theory Appl. 3, 583-594 (2009)

14. Davoodi, M.R., Golabi, A., Talebi, H.A., Momeni, H.R.: Simultaneous fault detection and control design for switched linear systems based on dynamic observer. Optim. Control Appl. Methods 34, 35-52 (2013)

15. Marcos, A., Balas, G.J.: A robust integrated controller/diagnosis aircraft application. Int. J. Robust Nonlinear Control 15 , $531-551$ (2005)

16. Li, T., Fu, J.: Event-triggered control of switched linear systems. J. Franklin Inst. (2017). https://doi.org/10.1016/j.jpranklin.2017.05.018

17. Shen, H., Su, L., Wu, Z., Park, J.H.: Reliable dissipative control for Markov jump systems using an event-triggered sampling information scheme. Nonlinear Anal. Hybrid Syst. 25, 41-59 (2017)

18. Wang, Y., Lim, C., Shi, P.: Adaptively adjusted event-triggering mechanism on fault detection for networked control systems. IEEE Trans. Cybern. 47, 2299-2311 (2017)

19. Shi, P., Wang, H., Lim, C.: Network-based event-triggered control for singular systems with quantizations. IEEE Trans. Ind. Electron. 63, 1230-1238 (2016)

20. Wang, Y., Shi, P., Lim, C., Liu, Y.: Event-triggered fault detection filter design for a continuous-time networked control system. IEEE Trans. Cybern. 46, 3414-3426 (2016)

21. Yue, D., Tian, E., Han, Q.: A delay system method for designing event-triggered controllers of networked control systems. IEEE Trans. Autom. Control 58, 475-481 (2013)

22. Amato, F., Ariola, M., Cosentino, C.: Finite-time stability of linear time-varying system: analysis and controller design. IEEE Trans. Autom. Control 55, 1003-1008 (2010)

23. Amato, F., Ariola, M., Cosentino, C.: Finite-time stabilization via dynamic output feedback. Automatica 42, 337-342 (2006)

24. Chen, J., Xiong, L., Wang, B., Yang, J.: Robust finite-time boundedness of $H_{\infty}$ filtering for switched systems with time-varying dely. Optim. Control Appl. Methods 37, 259-278 (2016)

25. Wen, J., Nguang, S.K., Shi, P., Peng, L.: Finite-time stabilization of Markovian jump delay systems-a switching control approach. Int. J. Robust Nonlinear Control 27, 298-318 (2017)

26. He, S., Liu, F.: Finite-time $H_{\infty}$ fuzzy control of nonlinear jump systems with time-delays via dynamic observer-based state feedback. IEEE Trans. Fuzzy Syst. 20, 605-614 (2012)

27. Wu, Y., Cao, J., Li, Q., Alsaedi, A., Alsaadi, F.E.: Finite-time synchronization of uncertain coupled switched neural networks under asynchronous switching. Neural Netw. 85, 128-139 (2017)

28. Lewis, F.L.: A survey of linear singular systems. Circuits Syst. Signal Process. 22, 3-36 (2007)

29. Zhao, Y., Zhang, W.: New results on stability of singular stochastic Markov jump systems with state-dependent noise. Int. J. Robust Nonlinear Control (2015). https://doi.org/10.1002/rnc.3401

30. Fu, L., Ma, Y.: Passive control for singular time-vary system with actuator saturation. Appl. Math. Comput. 289, 181-193 (2016)

31. Ma, Y., Yang, P., Yan, Y., Zhang, Q.: Robust observer-based passive control for uncertain singular time-delay systems subject to actuator saturation. ISA Trans. 67, 9-18 (2017)

32. Zhao, X., Zhang, L., Shi, P., Liu, M.: Stability of switched positive linear systems with average dwell time switching. Automatica 48, 1132-1137 (2012)

33. Wang, J., Ma, S., Zhang, C.: Stability analysis and stabilization for nonlinear continuous-time descriptor semi-Markov jump systems. Appl. Math. Comput. 279, 90-102 (2016) 
34. Liu, X., Yu, X., Zhou, X., Xi, H.: Finite-time $H_{\infty}$ control for linear systems with semi-Markovian switching. Nonlinear Dyn. 85, 2297-2308 (2016)

35. Yang, B., Wang, J., Wang, J.: Stability analysis of the delayed neural networks via a new integral inequality. Neural Netw. 88, 49-57 (2017)

36. Shen, H., Su, L., Park, J.H.: Further results on stochastic admissibility for singular Markov jump systems using a dissipative constrained condition. ISA Trans. 59, 65-71 (2015)

Submit your manuscript to a SpringerOpen ${ }^{\circ}$ journal and benefit from:

- Convenient online submission

$\checkmark$ Rigorous peer review

Open access: articles freely available online

- High visibility within the field

- Retaining the copyright to your article

Submit your next manuscript at $\boldsymbol{\Delta}$ springeropen.com 